This is the final peer-reviewed accepted manuscript of:

Bruno, F., Cameletti, M., Franco-Villoria, M., Greco, F., Ignaccolo, R., Ippoliti, L., Valentini, P., Ventrucci, M., 2016. A survey on ecological regression for health hazard associated with air pollution. Spat. Stat., 18, 276-299. https:// doi.org/10.1016/j.spasta.2016.05.003

The final published version is available online at:

https://doi.org/10.1016/i.spasta.2016.05.003

Rights / License:

The terms and conditions for the reuse of this version of the manuscript are specified in the publishing policy. For all terms of use and more information see the publisher's website. 


\title{
A Survey on Ecological Regression for Health Hazard Associated with Air Pollution
}

\author{
${ }_{3}$ Francesca Bruno $^{\mathrm{a}}$, Michela Cameletti ${ }^{\mathrm{b}}$, Maria Franco-Villoria ${ }^{\mathrm{c}}$, Fedele Greco ${ }^{\mathrm{a}}$, Rosaria \\ 5 \\ 6
}

\begin{abstract}
In the last 30 years, a large number of studies have provided substantial statistical evidence of the adverse health effects associated with air pollution. Statistical literature is very rich and includes a plethora of models to manage different types of spatial data. This paper starts with a thorough discussion on the spatial nature of the available data on health and air pollution. Health data are usually provided by Health Authorities as mortality and morbidity counts at a small area level. Thus we mainly focus on reviewing and discussing the spatial and spatio-temporal regression models proposed for disease count data on irregular lattices. In general, measuring the effect of exposure on health outcomes is an extremely hard task, and to obtain reliable estimates of the exposure effect and associated uncertainty one needs to build models that account for the residual variability not captured by the exposure-response relationship. In this context, Bayesian hierarchical models including spatial random effects play a prominent role: we consider both univariate and multivariate models and discuss some extensions to the spatio-temporal setting. Since model estimation can be prohibitive, practitioners are provided with a list of available software for Bayesian inference that avoids the need for complicated coding.
\end{abstract}

Keywords: Spatial and Spatio-Temporal Regression, Quantitative Health Risk Assessment, Change of Support Problem, Gaussian Markov Random Field, Hierarchical Bayesian Models, Factorial Models

\section{Introduction}

A substantial literature exists, starting from the late 1980s, on the adverse health effects associated with exposure to high levels of air pollution (see e.g. Schwartz and Marcus 1990; Dockery et al. 1993; Brunekreef and Holgate 2002; Schikowski et al. 2005; Lanki et al. 2006; Brook et al. 2010; Künzli 2012; Atkinson et al. 2014; Rushworth et al. 2014). This scientific evidence escalated at the end of 2013 with a study by the International Agency for Research on Cancer ${ }^{1}$ which found sufficient evidence to classify air pollution as a leading environmental cause of cancer deaths. The deleterious impact of air pollution on human health is also confirmed by the worldwide 3.7 million premature

\footnotetext{
${ }^{*}$ Corresponding author, email: rosaria.ignaccolo@unito.it

${ }^{1}$ https://www.iarc.fr/en/media-centre/iarcnews/pdf/pr221_E.pdf
} 
deaths attributable to ambient air pollution in 2012, an estimate provided by the World Health Organization (WHO) in $2014^{2}$. Air pollution is not only a major environmental risk to health but it represents also a societal cost: WHO reports that in 2010 the overall annual economic cost of health impacts and mortality from air pollution, including morbidity costs, amounted to 1.575 trillion US dollars for the countries of the WHO European region (WHO, 2015).

The range of adverse effects of ambient air pollution on health is wide, going from respiratory and cardiovascular diseases to hypertensive disorders and neurodegeneration, as documented by many studies. The impact of air pollution is known to be different depending on the length of the exposure periods, and a significant number of epidemiological studies have been conducted for investigating both the short and long-term effects of exposure. Starting from the 1990s, multicity time series studies have provided evidence of a strong association between short-term exposure to air pollution and adverse health events (in terms of mortality and morbidity). For example, the National Morbidity, Mortality, and Air Pollution Study (NMMAPS) in the US and the Air Pollution and Health: A European Approach (APHEA) study in Europe have found a $0.21 \%$ and a $0.6 \%$ increase in total mortality per 10 units elevation in particulate concentrations, respectively (see e.g. Samoli et al., 2003 and Peng et al., 2005). From the statistical point of view, this kind of associations are found by using Poisson time series regression models with the daily number of deaths/hospitalizations as outcome, the (possibly lagged) daily level of pollution as a linear predictor and smooth functions of weather variables and calendar time used to adjust for time-varying confounders (see e.g. Peng et al., 2006, 2009). There is also a vast literature that considers long-term air pollution exposure (see e.g. Carey et al., 2013; Cesaroni et al., 2013; Hoek et al., 2013; Lepeule et al., 2012): in this case data are collected through cohort studies and usually Cox proportional hazards models are used to investigate the associations between pollution concentrations and subsequent cause-specific mortality.

Recent advances in Geographical Information Systems (GIS) and global positioning systems enable accurate geocoding of locations where scientific data are collected. This has encouraged the formation of large monitoring networks to collect exposure measurements. Additionally, pollution concentrations estimated by computer dispersion models on a regular grid have also become widely available in recent times together with satellite measurements of remote sensing. These data sources, featured by a fine spatial resolution, have enabled researchers to examine relationships between disease rates for geographical areas and exposure to environmental risk factors using tools from spatial epidemiology (Elliott and Wartenberg, 2004). In general, health data are continuously collected by Health Authorities and usually consist of mortality and morbidity counts at the small area level, such as irregularly shaped administrative units. On the other hand, measurements of pollutants are associated with a set of monitoring stations and come in the form of geostatistical (or point-referenced) data.

In this review paper, we focus on these types of spatial data and provide a critical review of the statistical methods suggested for the analysis of aggregated count data in the context of ecological spatial and spatio-temporal regression. In particular, we discuss some important critical issues occurring in spatial epidemiology analysis and which typically are related to: $i$ ) the intrinsic features of the data, which are available from different sources and can be measured with error and with different spatial resolutions; ii) the specification of a regression function that typically relates a change in air pollution to health disease risk; iii) the acknowledgement of spatial and temporal dependence in regression models, together with potential biases and confounding; and $i v$ ) the characterization of uncertainty for risk estimates. Other review papers about spatial analysis in environmental epidemiology exist (see e.g. Jerrett et al., 2010; Robertson et al., 2010; Carpenter, 2011; Meliker and Sloan, 2011; Osei, 2014), but most of them are devoted to single specific topics (e.g. clustering methods, surveillance methods, GIS) and keep a very general overview of the related statistical critical issues. On the other hand, the slant of our review paper is on the statistical aspects of data collection and analysis, and it provides a deeper insight into ecological spatial and spatio-temporal regression, while trying to be simple in the notation in order to be readable by a large community of scientists and researchers including geographers, epidemiologists, etc.

The rest of the paper is structured as follows. Section 2 introduces data commonly used in health and air pollution studies and discusses the most relevant issues, including exposure metric, spatial misalignment, preferential sampling, measurement error and ecological fallacy. Ecological spatial and spatio-temporal regression models are described in Section 3, with a detailed discussion on spatial random effects and multivariate models required in the case of multiple disease data. A short review of the available software for the considered models is given in Section 4, while discussion points are given in Section 5.

\footnotetext{
${ }^{2}$ http://www . who.int/mediacentre/news/releases/2014/air-pollution/en/
} 


\section{Data in health and air pollution studies}

Concerns about the health effects of air pollution from industrial, traffic and heating sources are currently the focus of considerable public and scientific attention. In general, the modelling approach used for assessing the impact of exposure on human health depends on the kind of data available and how the data are collected over space and/or time. Data used in studies to evaluate the exposure-risk relationship usually include disease events, measurements on pollutants or other risk factors and the reference population at risk, along with other covariates describing sociodemographic features. Air pollution concentrations are routinely measured at a number of monitoring sites across a region of interest producing point-reference data or are simulated by numerical models over a pre-set grid of locations. On the other hand, health data are mostly collected at an aggregated level and are usually associated with irregularlyshaped (administrative) regions. Epidemiological investigations in which associations between disease occurrence and environmental risk factors are studied over aggregated groups rather than on individual level are termed ecological studies. Even if these studies have been questioned because of their aggregated nature, they are particularly useful when individual level measurements of exposure are not available, as in case studies concerned with the investigation of the effect of air pollution on human health (Biggeri et al., 2004; Samet et al., 2000; Smith et al., 2000). This section provides a brief background on the kind of data available in air pollution health related studies and describes the potential problems of working with these types of data.

\subsection{Health data}

In statistical epidemiology, health data have usually been studied either in the form of count data or at individual level (case event data). In the latter case, two main types of studies can be distinguished: those based on analyzing the spatial pattern of case event locations using point processes (Grell et al., 2015; Diggle, 1990, Diggle, 2003, Pinto Junior et al., 2015), and cohort studies, which are useful to quantify the health effects resulting from long-term exposure (months or years) to pollution (see e.g. Molitor et al., 2006; Hoek et al., 2013). Firstly, they are not easily available and are costly to obtain due to the large amount of data required and the possible expenses related to GIS and global positioning system (GPS)-based devices (Nuckols et al., 2004). Secondly, the case home address could not be related to the disease aetiology. For instance, the disease could have been contracted in the working address because of occupational exposure or could be related to the time spent by individuals in several indoor and outdoor microenvironments characterized by different pollutant concentrations. In this regard some authors have proposed models for personal exposure based on longitudinal panel studies, time-activity diary data or GPS-enabled individual exposure monitor, see e.g. McBride et al. (2007), Zidek et al. (2007) and Blangiardo et al. (2011). As a result of these drawbacks related to individual data, either time series or areal unit study designs have also been used to quantify the short- and long-term health impact of air pollution. These studies utilise population level summaries rather than individual level data and cannot be used to quantify individual level cause and effect. However, both time series and areal unit (aggregated) data required to implement such studies are routinely collected by Health Authorities and are widely available. Although several studies have been proposed using both time series (Peng and Dominici, 2008) and spatial designs, here we only focus on areal data and the related spatial and spatio-temporal modelling challenges.

Health data are collected over time in a fixed study region, $\mathcal{D}_{y}$, typically in the form of mortality and morbidity counts or hospital admissions, coded according to the type of disease (e.g. cardiovascular, acute respiratory, etc). In general, occurrences of several, say $n_{y}$, diseases can be observed at a specific region (e.g. zip codes, counties), $\mathbf{s}_{i}$, and at a specific time point $t$. The complete set of information for the health data is thus denoted as

$$
\left\{Y_{k}\left(\mathbf{s}_{i}, t\right), \mathbf{s}_{i} \in \mathcal{D}_{y}\right\}, \quad k=1, \ldots, n_{y} ; i=1, \ldots, N_{y} ; t=1, \ldots, T
$$

where $k$ and $i$ are the disease and region index, respectively. A great advantage of working with count data is their quick availability for a wide variety of diseases, particularly in countries with advanced statistical systems. This allows studying many variables and populations at relatively low cost (with respect to cases when data are not routinely collected). Because of its quick availability, this kind of data has been object of considerable methodological developments in the last years (see Section 3).

However, there are a number of problems than can arise from aggregating data into geographic units, including small number problem and frequent zero-valued observations (when events are counted in a short period of time and/or on very small areas for a rare disease; see Section 3.1.3), modifiable area unit problem (MAUP) and ecological 
fallacy (see Section 2.3), and changes in geographic unit boundaries over time (Waller and Gotway, 2004; Meliker and Sloan, 2011).

\subsection{Air pollution data}

Air pollution data are normally used as a measure of exposure in spatial and spatio-temporal regression models to evaluate the associated health risk. Pollution concentrations are routinely measured at specific points in time and at a number of monitoring sites across a continuous region $\mathcal{D}_{x}$ and usually come in the form of geostatistical data. In general, one or more pollutants can be observed at each monitoring site so that the entire set of information for these data, at a specific monitoring site $\mathbf{u}_{l}$ and a specific time point $t$, is denoted by

$$
\left\{X_{j}\left(\mathbf{u}_{l}, t\right), \mathbf{u}_{l} \in \mathcal{D}_{x_{j}}\right\}, \quad j=1, \ldots, n_{x} ; l=1, \ldots, N_{x_{j}} ; t=1, \ldots, T ;
$$

\footnotetext{
${ }^{3}$ http://www.epa.gov/air-research/community-multi-scale-air-quality-cmaq-modeling-system-air-quality-management

${ }^{4}$ http://www.Imd.polytechnique.fr/chimere/
} 
Another choice to be made regarding the metric concerns the identification of suitable summary indicators (e.g. maximum, average or other syntheses of pollution concentration). Huang et al. (2015) evaluated the impact of the chosen summary on the estimated exposure effect $\left(\mathrm{NO}_{2}\right)$ on respiratory disease in Scotland over the period 2007-2011 and found that only maximum $\mathrm{NO}_{2}$ (within each areal unit) appears to have a significant effect on the relative risk of respiratory disease, while the area average does not.

In the literature there are also some works which take into account that air pollution is a heterogeneous mix of several compounds of different size, source and toxicity. For example Atkinson et al. (2010) and Pirani et al. (2015) consider $\mathrm{PM}_{10}$ and $\mathrm{PM}_{2.5}$ concentration together with particle number concentration and composition (carbon, sulfate, nitrate and chloride) for an epidemiological time series study. The limitation is that this kind of particle metrics are usually collected at a limited number of monitoring sites and it is difficult to include a spatial dimension in the analysis.

\subsubsection{Spatial misalignment and Change of Support Problem (COSP)}

In this paper we refer to spatially misaligned data as data measured on different spatial supports (i.e. at point or area level). For this reason, it is important to deliver statistical methods that are able to cope with different spatial resolutions in order to effectively change the support of air pollution data (regressors) to achieve alignment with the outcome measured at area level. Disease data appear as counts observed at irregularly-shaped regions $\mathbf{s}_{i} \in \mathcal{D}_{y}$. In contrast, pollution data are typically available at points $\mathbf{u}_{l}$ within a study region $\mathcal{D}_{x_{j}}$ and appear irregularly spaced. This spatial misalignment gives rise to the Change of Support Problem (COSP) which refers to the issues deriving from making inference with data on different spatial supports (point or area level) and resolutions (e.g. zip, tract, district, grid cell, pixel, etc.). As reviewed in Gotway and Young (2002) and Gelfand et al. (2010), COSP is a generic term which encloses many cases as well as several solutions and approaches.

In spatial epidemiology, the COSP case consists in upscaling from point to area level. Usually, a two stage approach is adopted: the first stage predicts pollution exposure for each region $\mathbf{s}_{i}\left(i=1, \ldots, N_{y}\right)$ using air pollution concentrations $X_{j}\left(\mathbf{u}_{l}\right)$ available at the monitoring sites $\mathbf{u}_{l}, l=1, \ldots, N_{x_{j}}$; in the second stage these predictions are linked with the health outcomes through a spatial regression model (see Section 3 ).

At the first stage a naive approach can be implemented and consists in computing the area-wide pollutant level as the average of the values measured by the neighboring monitoring station(s), possibly using distance- or population-based weights (Elliott et al., 2007; Madsen et al., 2008). This approach is reasonable for pollutants with a spatial homogeneous behavior but fails in representing the true concentration distribution especially when the number of monitors is limited and they are not randomly placed throughout the considered area (Lee et al., 2015), or when particulate matter constituents, which show a strong spatial heterogeneity, are taken into account (Krall et al., 2015). In these cases the pollutant values measured at a single or limited number of monitors cannot be considered as an accurate estimate of the average pollutant level across the area. When pollutant concentrations are available on a regular grid via numerical air quality and atmospheric dispersion model, the realignment with the health data can be simply obtained by computing an average over the intersections between the areas and the regular grid cells (see e.g. Rushworth et al., 2014; Lee and Sarran, 2015).

An alternative consists in implementing a spatial model for the measured pollutant values in order to predict the concentrations at some unobserved locations, and then average these predictions to obtain the exposure level over the areas. In particular, point predictions can be obtained via geostatistical kriging, interpolation by inverse distance weighting (e.g. Carlin et al., 1999; Hubbell et al., 2005; Bell, 2006; Young et al., 2009; Li et al., 2014), or via land use regression (Hoek et al., 2008; Basagaña et al., 2013). A similar spatial prediction technique for point-block realignment is block kriging (Cressie, 1993), which is a predictor of the average value over a region $\mathbf{s}_{i} \in \mathcal{D}_{y}$. Zhu et al. (2003) present a Bayesian application of spatial upscaling based on block kriging implemented through Monte Carlo approximation of integrals over grid points.

Even if easy and intuitive these approaches may not be able to provide an accurate estimate of the exposure distribution. Recently a different line of research has been explored in several papers which consider the true pollution level as a latent spatial (or spatio-temporal) continuos process, measured with error at a finite number of sites, and defined through a stochastic model possibly including spatially varying covariates (e.g. meteorological variables) or temporal dynamics. Furthermore, in a data fusion perspective, it is also possible to merge data coming from air quality numerical models or remote sensing observations. This approach is adopted for example by Wikle and Berliner (2005), Fuentes and Raftery (2005), Fuentes et al. (2006), Choi et al. (2009), Peng and Bell (2010), Lawson et al. (2012), Huang et al. (2015) and Blangiardo et al. (2016), with the possibility of obtaining the area level pollutant con- 
centrations by averaging estimates available by sampling from the posterior predictive distribution at several locations chosen within an area on a given time point. All these aggregation and upscaling approaches provide area-wide exposure estimates which are usually plugged-in the linear predictor of the second stage health model (see Equation (1)) by assuming they are known covariates. This approach fails to properly consider the exposure uncertainty, possibly producing a biased estimation of air pollution risk on the health outcome. A first solution to this issue is the fully Bayesian model proposed by Lee and Shaddick (2010), which feeds the entire pollutant concentration posterior predictive distributions into the second stage, thus accounting for the uncertainty intrinsic in the concentration estimates. Alternatively, a measurement error approach can be adopted, like the one proposed for example by Gryparis et al. (2009) and Szpiro and Paciorek (2013) based on a Berkson error model which allows to reduce bias in the second stage estimation (health model). The Bayesian Maximum Entropy (BME) method is an alternative proposed by Lee et al. (2009) to deal with the COSP thus providing a framework for the non-linear integration of data obtained at different observation scales.

\subsubsection{Preferential sampling}

Typically, exposure is assessed using data from an existing monitoring network. However, air quality monitoring stations may be intentionally located in certain places; in many cases, they are located close to important sources of pollution, with the aim of monitoring compliance with air pollution regulation, resulting in overestimated exposure. When exposure is estimated at the area level, the assumption is that observed locations were selected at random, leading to inaccurate estimation of exposure (Shaddick and Zidek, 2014; Zidek et al., 2014) and hence, of its effect on health (Lee et al., 2015). Shaddick and Zidek (2014) show evidence of the effect of preferential sampling when assessing exposure. They found statistically significant differences in terms of pollution levels between stations within a monitoring network in the UK that were kept during the whole time period the network was working and stations that at some point were removed; i.e. stations at which pollution levels are low tend to be deleted from the network. Zidek et al. (2014) propose a general framework using a superpopulation modelling approach to adjust exposure estimates for preferential sampling. Lee et al. (2015) investigate the effect of preferential sampling on health effect estimates in a two-stage model via a simulation study and using real data; exposure is modelled at the first stage using data from a preferentially sampled network and from a randomly sampled network, leading to different conclusions about the effect of exposure on health, as well as having an effect on the variability and bias of the estimated effect.

\subsubsection{Measurement error}

To assess the effect of air pollution on the human health, accurate exposure measurements or estimates are necessary. Since it is usually not possible to measure individual exposure, a concentration field over an area at a certain time is considered as a surrogate. However this field can be measured only through a monitoring network providing measures at fixed locations with a certain time frequency. Thus a measurement error can occur, that can be due to instrument imprecision at a monitoring site as well as to prediction error of the model applied to obtain a concentration value at an unmonitored site. Moreover, when the spatial support of health data does not coincide with that of air pollutant data, the COSP arises and the misalignment leads to an error that can be taken into account in a proper model (see also Sections 2.2.2 and 3.1.1). Also, concentration fields may be often obtained as output of numerical models that are affected by uncertainty related to initial conditions, parameters in model equations as well as model structure (Bayarri et al., 2009; Paci et al., 2015); such uncertainty constitutes a calibration error that could propagate to the response when model output is used as a predictor. To take into account this error and combine numerical output with observations, the data fusion and data assimilation techniques have been developed (Gelfand et al., 2010; Evensen, 2009). Data fusion consists in merging data coming from various sources of different nature and in the spatial context it requests to deal with COSP (see Section 2.2.2). Instead, data assimilation, which can be seen as a particular case of data fusion, deals with the inclusion of observed data into numerical models to estimate initial conditions in a predictive model consistent with the available observations and the underlying model dynamics.

In general, to include measurement errors the so-called error-in-variables (EIV) models are considered, often referred to as measurement error models (MEM); for a general overview see Fuller (1987) for linear models and Carroll et al. (2006) for nonlinear ones. Two types of MEM are distinguishable: the classical error model and the Berkson error model (Shaddick and Zidek, 2015). Under the first one, the measurement model equation states that the observed exposure is randomly distributed around the true latent level in the additive version (see e.g. Van de Kassteele and Stein, 2006). Instead the Berkson error model specifies the distribution of the true unobserved exposure 

as being dependent on the observed exposure measurement. Both models can be additive or multiplicative, and a spatial component can be incorporated in the model error term (Gray et al., 2011).

For spatial exposure, some works (Gryparis et al., 2009; Szpiro et al., 2011; Sheppard et al., 2012; Szpiro and Paciorek, 2013) decompose the measurement error into a Berkson-like component from smoothing the exposure surface and a classical-like component from variability in estimating exposure model parameters, even considering spatial misaligned data (see also Section 2.2.2).

\subsection{Ecological Fallacy and MAUP}

Inferences about individuals at locations are functions of regional average characteristics that are ascribed to a particular point in space and to a particular individual; assuming that associations observed at area level hold for the individuals within the areas can lead to the so-called ecological fallacy (Piantadosi et al., 1988; Greenland and Morgenstern, 1989; Greenland and Robins, 1994; Clayton et al., 1993; Morgenstern, 1998; Wakefield and Elliot, 1999; Wakefield, 2003). Several works have investigated the implications of ecological fallacy and the inherent bias under a modelling perspective (see Wakefield and Lyons (2010) and references therein). Given a non-linear model for the individual risks, such model changes when individual risks are aggregated at the areal level (specification bias). Wakefield and Shaddick (2006) use this modelling framework to show that specification bias is small if the within area variability in exposure is close to zero. They also point out that "the key to minimizing ecological bias is to have a fine enough partition of space at which exposure measurements are available, relative to the spatial exposure variability". As discussed by Wakefield and Lyons (2010), the only way of accounting for the loss of information implied by working with areal data (and therefore alleviate the ecological fallacy problem) is to include individual level data in the modelling process. In particular, Jackson et al. (2008) proposed what they have termed as hierarchical related regression to jointly model aggregated and individual data, improving ecological inference.

Inference based on count data is subject to bias due to their aggregate nature. This is a problem found in nonspatial epidemiological studies (see for example Breslow and Day, 1980) and is amplified in spatial studies, where the effect of aggregation on spatial correlation constitutes an additional source of bias. From the spatial point of view, ecological fallacy arises because inference may change depending on how data are aggregated into geographical regions. The spatial manifestation of ecological fallacy has been recognised as a particular case of the Modifiable Areal Unit Problem (MAUP, Gotway and Young, 2002), which comprises two interrelated problems: the scale effect and the zoning effect (Openshaw and Taylor, 1981). In the former, inference changes when data are grouped into increasingly large geographical regions; this is the analogous of aggregation bias in non-spatial ecological studies arising when individuals are grouped. The source of this problem is the smoothing effect resulting from averaging, which reduces heterogeneity among units, or equivalently, some information about the spatial variability of the cases is lost. Aggregation-induced bias depends on the heterogeneity in the grouped observations: a completely homogeneous grouping system would be free from this problem (Openshaw, 1984). Indeed, as first observed in the pioneering work of Roberston (1950), the correlation between two variables measured at an ecological level can be expressed as the sum of the within group and between group components; when the within group component is negligible the ecological bias vanishes. This means that the smoothing effect is alleviated if the original observations are characterised by positive spatial correlation. On the other hand, the zoning effect arises when alternative formations of the areal units lead to different results; if the variation among areal units is not constant over the study region, different zoning rules can lead to different spatial correlation structures. This is the analogous of the grouping effect in non-spatial ecological studies.

\section{Spatial and spatio-temporal regression}

The association between air pollution and health data has been typically studied through the development of three-level hierarchical regression models. Such models have an intuitive appeal and enjoy several advantages. For example, they are well-suited for incorporating the foregoing knowledge at various levels of the modelling, are easy to interpret and facilitate model fitting. Furthermore, within the Bayesian paradigm, they enable exact inference and proper uncertainty assessment within the given specification.

In general, at the first level, the health data are conditioned on the process and parameters. The data can be conditioned on whatever aspects of the process are appropriate. At the second level of the hierarchy the process 
component is specified. This component can have multiple levels, it can be spatial and/or dynamic and its stochastic form can be univariate or multivariate. Finally, at the third level, hyperprior distributions are specified (Gelfand, 2012).

In this section, we start by critically reviewing procedures for prior choices used in ecological spatial regression models for count data and then extend the study to spatio-temporal regression. These models are typically developed for modelling single diseases. However, in many cases, joint modelling of diseases can increase the understanding of diseases dynamics and relationships among diseases incidence. The merit of joint modelling can be high if the considered diseases share environmental risk factors. This has led to the development of multivariate spatial methods suited for areal data. The last part of the Section is thus dedicated to the discussion of multivariate spatial regression and the specification of spatial and spatially dynamic factor models, followed by a brief discussion on spatio-temporal regression models.

\subsection{Univariate spatial regression}

Suppose that the region of interest, $\mathcal{D}_{y}$, is split into $N_{y}$ contiguous areas and that the observed number of disease cases in an area is denoted as $Y\left(\mathbf{s}_{i}\right)$, with $\mathbf{s}_{i} \in \mathcal{D}_{y}$. To assess which areas exhibit elevated or low levels of disease risk, the number of cases expected to occur in each area, $E\left(\mathbf{s}_{i}\right)$, is calculated; expected counts are thought as fixed and known functions of the size and demographic structure of the population living within each area (Banerjee et al., 2004; Lawson, 2009).

The number of expected cases (calculated using internal standardization) is what would be observed if the disease risk was constant over the whole study region and the spatial variations in incidence were due uniquely to population density and structure. In disease mapping studies, the focus is on identifying features of the spatial distribution of the disease rate that depart from what expected under the assumption of a constant disease risk.

If $E\left(\mathbf{s}_{i}\right)$ is not too large (i.e. the disease is rare and/or the population at risk is small), a standard univariate spatial model for the $Y\left(\mathbf{s}_{i}\right)$ is given by the Poisson model (Lawson, 2009)

$$
Y\left(\mathbf{s}_{i}\right) \mid \eta\left(\mathbf{s}_{i}\right) \stackrel{i n d}{\sim} \operatorname{Po}\left(E\left(\mathbf{s}_{i}\right) e^{\eta\left(\mathbf{s}_{i}\right)}\right)
$$

with $\eta\left(\mathbf{s}_{i}\right)$ being the log relative risk in areal unit $\mathbf{s}_{i}$. This model completes with the linear predictor

$$
\eta\left(\mathbf{s}_{i}\right)=\mu\left(\mathbf{s}_{i}\right)+\phi\left(\mathbf{s}_{i}\right)
$$

where $\mu\left(\mathbf{s}_{i}\right)$ is a mean level component and $\phi\left(\mathbf{s}_{i}\right)$ is a random effect introduced to capture any residual spatial autocorrelation present in the data.

\subsubsection{Modelling the mean component}

The simplest specification of the mean component appears as a parameterised function of the type, $\mu\left(\mathbf{s}_{i}\right)=\tilde{\boldsymbol{x}}\left(\mathbf{s}_{i}\right)^{\prime} \boldsymbol{\beta}$. The associated vector of regression coefficients, $\boldsymbol{\beta}$, is of main interest and helps in evaluating the impact of exposure variables on the health outcome under examination. Because several sources of uncertainty are in place, several statistical issues must be considered when trying to reliably estimate $\beta$.

Vector $\tilde{\boldsymbol{x}}\left(\mathbf{s}_{i}\right)$ denotes exposure variables at area $\mathbf{s}_{i}$. Usually, exposure variables are not directly observed at area level and the pollution concentrations must be estimated on administrative regions by applying COSP methods as discussed in Section 2.2.2. Also, the Poisson log-linear model considers the vector of estimated pollution concentrations as known and assumed to be constant across each areal unit. The hierarchical modelling approach allows to address the misalignment between health and exposure data in a straightforward manner, by simply adding a further level for Bayesian spatial prediction to estimate the pollution concentrations for all the areal units. The specification of a measurement error model for the exposure also allows to treat such exposure variables as model parameters and fully Bayesian inference guarantees that uncertainty concerning the parameters defined at lower levels correctly propagates through the hierarchy, giving proper assessment of the uncertainty for $\beta$ at the first level.

There are also two major difficulties in making inference on regression parameters. Firstly, exposure variables in $\tilde{\boldsymbol{x}}\left(\mathbf{s}_{i}\right)$ might be themselves highly correlated, leading to multicollinearity. There exist several broad strategies to deal with multicollinearity and typical examples are represented by ridge regression and partial least squares regression (Brown, 1993). Another possibility is to perform a dimensionality reduction procedure on exposure variables by means of principal components or factor analysis; the latter is discussed in Section 3.2.2. 
A second issue regards allowance for the effect of confounders into the model. A variable is a confounding factor for the effect of air pollution on health when it is correlated with both the health outcome and the associated exposure variables. For a formal definition of confounding in epidemiological studies and possible solutions to deal with it see, for example, Rothman et al. (2012). Typical confounders considered in the literature include age, gender, ethnicity and socio-economic deprivation. Meteorological variables, such as temperature and humidity, are also well known to be confounders when analysing the relationship between air pollution and mortality, especially in short-term effect studies. Not accounting for all potential confounders may cause severe bias in estimating $\boldsymbol{\beta}$ and can lead to wrong conclusions on the effect of exposure on health (Shaddick and Zidek, 2015).

It is important to distinguish between unmeasured and measured confounders (Peng et al., 2006). When confounding variables are measured, standard practice is to include them into the mean component in model (1), so that $\mu\left(\mathbf{s}_{i}\right)=\tilde{\boldsymbol{x}}\left(\mathbf{s}_{i}\right)^{\prime} \boldsymbol{\beta}+\tilde{z}_{\boldsymbol{m}}\left(\mathbf{s}_{i}\right)^{\prime} \boldsymbol{\beta}_{z}$, where $\tilde{z}_{\boldsymbol{m}}\left(\mathbf{s}_{i}\right)$ is the vector of measured confounders at areal unit $\mathbf{s}_{i}$ and $\boldsymbol{\beta}_{z}$ is the associated vector of parameters.

Managing the effect of unmeasured confounders is a more controversial task to be addressed. A popular approach is to assume that these unmeasured confounders drive the residual spatial correlation, after accounting for exposure and measured confounders in the mean component, and that this variability can be captured by the spatial random effects, $\phi\left(\mathbf{s}_{i}\right)$, in model (1). Hence, omitting the random effects, $\phi\left(\mathbf{s}_{i}\right)$, from the model is not a sensible choice as its dependence with the covariates causes bias in estimating $\boldsymbol{\beta}$.

\subsubsection{Modelling the spatial random effect}

Since the random variables $\phi\left(\mathbf{s}_{i}\right)$ in equation (1) are devoted to capture area-level spatial effects that are representative of a whole region, models suited for lattice data, i.e. Gaussian Markov Random Fields (GMRF, see Rue and Held, 2005 for a comprehensive overview), arise as a natural choice in this context and are almost the standard in the literature concerning ecological spatial regression. For this reason, they receive major attention in this Section. Alternatively, an example of a geostatistical approach is given in Kelsall and Wakefield (2002), where the correlation structure is derived through consideration of an underlying continuous risk surface. The multivariate Normal distribution is one of the most flexible distributions for representing spatially correlated random variables. For instance, writing $\phi=\left(\phi\left(\mathbf{s}_{1}\right), \ldots, \phi\left(\mathbf{s}_{N_{y}}\right)\right)$, one might assume that $\phi \mid \varphi \sim N(\mathbf{0}, \boldsymbol{\Sigma}(\varphi))$, where $\boldsymbol{\Sigma}(\boldsymbol{\varphi})_{i j}$ gives the covariance between $\phi\left(\mathbf{s}_{i}\right)$ and $\phi\left(\mathbf{s}_{j}\right)$ as a function of some hyperparameters $\varphi$ (Banerjee et al., 2004).

However, in health care research, there is rarely much substantive knowledge to guide the choice of the covariance function, and often quite weak information in the data to estimate the parameters of this function, particularly for more complex forms. As high long range correlation of the risks is difficult to distinguish from the effect of the overall mean, it is also important to ensure that the chosen correlation function (and associated hyperpriors) gives near zero correlation at distances within the extent of the study region, to avoid nonidentifiability of the mean and correlation parameters (Best et al., 2005). Moreover, a further important practical limitation of this approach is that with large study regions the implementation via Markov chain Monte Carlo (MCMC) algorithms can be computationally expensive due to the large amount of matrix inversion required at each iteration.

As stated before, the most relevant class of models in the context of regression involving an area-level response is that of GMRFs, also known as conditional autoregressive (CAR) models (see, e.g., Cressie, 1993, sec. 6.3.2). These models specify conditional dependence involving a (usually small) set of spatial neighbours. The specification of the neighbourhood structure is commonly based on the adjacency matrix $\mathbf{W}$, which is a symmetric $0 / 1$ matrix with element $w_{i j}$ equal to 1 if $i$ and $j$ are neighbours (by definition, $w_{i i}=0$ ). CAR models are specified starting by $N_{y}$ conditional distributions:

$$
E\left(\phi\left(\mathbf{s}_{i}\right) \mid \phi\left(-\mathbf{s}_{i}\right)\right)=\sum_{j} \alpha_{i j} \phi\left(\mathbf{s}_{j}\right), \quad \operatorname{Var}\left(\phi\left(\mathbf{s}_{i}\right) \mid \phi\left(-\mathbf{s}_{i}\right)\right)=\kappa\left(\mathbf{s}_{i}\right),
$$

where $\phi\left(-\mathbf{s}_{i}\right)$ denotes all other values but $\phi\left(\mathbf{s}_{i}\right), \alpha_{i j}=\rho w_{i j} / w_{i+}$, with $w_{i+}=\sum_{j} w_{i j}$, and $k\left(\mathbf{s}_{i}\right)=\tau^{2} / w_{i+}$. The $\alpha_{i j}$ are coefficients reflecting local spatial dependence between units $i$ and $j$ while $k\left(\mathbf{s}_{i}\right)$ is the conditional variance which is inversely proportional to the number of neighbours. Note that this specification for $\alpha_{i j}$ and $\kappa\left(\mathbf{s}_{i}\right)$ satisfies the symmetry condition, $\alpha_{i j} k\left(\mathbf{s}_{j}\right)=\alpha_{j i} K\left(\mathbf{s}_{i}\right)$, which is a necessary condition for the joint distribution of $\boldsymbol{\phi}$ to be valid (Cressie, 1993).

The parameter $\rho$ can be thought of as an autocorrelation parameter that reflects the overall strength of spatial dependence between locations with nonzero weights. To define a proper joint distribution, the covariance (or the 
precision) matrix must also be positive definite: this requires that $|\rho|<1$ if the scaled adjacency matrix, $\boldsymbol{D}^{-1} \mathbf{W}$, where $\boldsymbol{D}=\operatorname{diag}\left(w_{i+}\right)$, is used (Cressie, 1993).

An appealing feature of the GMRF prior is the possibility to make inference about the overall degree of spatial dependence in the disease risk by estimating $\rho$. However, interpretation of $\rho$ is not straightforward and values close to the maximum are needed to reflect even moderate spatial dependence. This drawback led Besag et al. (1991) to consider the intrinsic CAR model as a prior for $\phi$. This model is obtained by setting $\rho$ to its upper limiting value of 1 such that the conditional expectations for $\phi\left(\mathbf{s}_{i}\right)$ are equal to the mean of the random effects in neighbouring areas. This is the simplest possible CAR prior and is appropriate if the residuals from the covariate component of the linear predictor are spatially smooth across the entire region. Also, although the univariate conditional prior distributions are well defined, the corresponding joint multivariate Gaussian distribution of the intrinsic CAR is improper since the precision matrix is singular. Finally, notice that the improper CAR prior is a pairwise difference prior that can be identified only up to an additive constant. Hence, to identify an intercept term in the linear predictor, a sum-to-zero constraint on the random effects is needed (Banerjee et al., 2004).

The instrinsic CAR model is suited for capturing unexplained spatially structured variability. In order to capture both spatially structured and unstructured variability, Besag et al. (1991) proposed to introduce a second set of independent Gaussian random effects, say $v\left(\mathbf{s}_{i}\right)$, with mean zero and a common variance. Following this model specification, which is also known as the Besag-York-Mollié (BYM) or convolution model, different levels of spatial smoothness can be achieved by varying the relative sizes of the two components $\phi\left(\mathbf{s}_{i}\right)$ and $v\left(\mathbf{s}_{i}\right)$. However, the disadvantage of this flexibility is that each data point is represented by two random effects, and hence only their sum is identifiable. A thorough discussion about difficulties concerning model estimation and identifiability of area-specific random effects $\phi\left(\mathbf{s}_{i}\right)$ and $v\left(\mathbf{s}_{i}\right)$ is provided in Eberly and Carlin (2000).

Alternatives to the convolution model, which avoid the potential identifiability problem encountered with the BYM prior, have been proposed by MacNab (2003) and Stern and Cressie (2000). However, these extensions consider a global smoothing parameter, such that the amount of smoothing performed is affected globally by all the areas and is not adaptive. This could be inappropriate for two reasons. Firstly, since the spatial distribution of air pollutant is smooth, the residual spatial structure obtained after considering the covariate effect is unlikely to be globally spatially smooth, and is instead likely to exhibit localised smoothness (Lee et al., 2014). Secondly, if the spatial random effects capture the effect of spatially correlated unmeasured variables, potential collinearity between this component and any other spatially smooth covariate can lead to variance inflation and bias in the estimation of the air pollution effect, essentially because of spatial confounding (Hodges and Reich, 2010; Paciorek, 2010). As discussed by Paciorek (2010), the size of the spatial confounding bias depends on the spatial scale of the variability in the outcome and the exposure and can only be reduced if the unmeasured confounders (in the form of spatial random effects) are responsible for the large-scale spatial variability in the outcome, with exposure only explaining the small-scale spatial patterns.

To our knowledge, approaches to solving these problems have been proposed either by spatially constrained regression, where the spatial random effects are orthogonal to the covariates (see, for example, Reich et al., 2006, Hodges and Reich, 2010, and Hughes and Haran, 2013), or relaxing the global smoothing restrictions of the CAR prior to allow for localized priors as discussed in Lee et al. (2014). Other authors have also developed semiparametric spatial models, that replace the continuously varying spatial distribution for the log-relative risk by discrete allocation or partition models with each cluster or component having a constant unknown relative risk. A critical discussion of this approach can be found in Best et al. (2005).

\subsubsection{Departures from the Poisson model}

The spatial epidemiological literature often refers to extra-Poisson variability in the data to indicate any departure from the Poisson model (i.e. variance equal to the mean). The most frequent situation is overdispersion, which is when the health outcome has variance larger than the mean. The introduction of the spatial random effects $\phi\left(\mathbf{s}_{i}\right)$ in (1) can be seen as a device for inducing over-dispersion, or extra-Poisson variation, into the model. More precisely, $\phi\left(\mathbf{s}_{i}\right)$ captures extra-Poisson variation due to spatial correlation (which may be induced by unobserved confounders varying at the areal level, e.g. socio-economic factors; see also discussion in Section 3.1.1). Extra-Poisson variability can also be driven by spatially unstructured unobserved confounders, i.e. varying within areas at an individual level (e.g. smoking), or simply be caused by anomalies in the data (Wakefield and Elliot, 1999). In general, models which fail to account for overdispersion lead to underestimation of the uncertainty associated to the estimates of the log 
relative risks $\eta\left(\mathbf{s}_{i}\right)$ (Shaddick and Zidek, 2015). A popular model for overdispersed counts is the negative binomial distribution, i.e. a Poisson with a Gamma distribution on the mean parameter, which offers a suitable approach to deal with spatially unstructured extra-Poisson variability in the data. Based on a Poisson-Gamma framework, Wakefield (2007) investigates several parametrizations for the Gamma distribution giving different variance mean relationships. The framework for regression presented throughout Section 3 allows to accommodate spatial extra-Poisson variability by means of area-level random effects in a Poisson log-Normal model. A more general approach, which is able to cope both with under and overdispersion, consists in using a Generalised Poisson Likelihood, see Fuentes et al. (2006) for an application of this model in evaluating the effect of particulate matter pollution on health.

When dealing with a data set with an excessive number of zeros, zero-inflated models can be used. The zero inflated Poisson (Agarwal et al., 2002) model has received a lot of attention and has always been used in modelling count data where the extra variations are solely caused by the extra zeros. For health data where the extra variability is caused by excess zeros and also unobserved heterogeneity, recommended models may be, for example, zero inflated negative binomial and zero inflated generalized Poisson (Gschlößl and Czado, 2008).

\subsection{Multivariate extensions}

Many health care research studies refer to the joint study of multiple diseases. In general, the abundance of measures is both an opportunity and a challenge from the epidemiological and the statistical point of view. From the epidemiological point of view, joint modelling of diseases can increase the understanding of diseases dynamics and relationships among diseases occurrence. The merit of joint modelling can be high if the considered diseases share risk factors or if the presence of a disease encourages (or inhibits) the occurrence of another one. From the statistical point of view, an evident advantage in joint disease mapping is that, if the disease risks are correlated, standard errors of the estimates obtained via univariate modelling can be sensibly reduced. Moreover, estimates for rare diseases can borrow strength from more diffuse diseases. In fact, correlation between diseases within areas, between areas within diseases and between areas and diseases constitutes valuable information contained in the data that can be used to increase efficiency of the estimates.

Modelling several diseases jointly in an effective way, thereby borrowing information across them, is a difficult task in general. While models for univariate disease data have been extensively explored, models for multivariate lattice data have only been developed in the past few years.

Let $Y_{k}\left(\mathbf{s}_{i}\right)$ and $E_{k}\left(\mathbf{s}_{i}\right)$ denote the observed and expected number of cases for disease $k=1, \ldots, n_{y}$ in a region $\mathbf{s}_{i}$, with $\mathbf{s}_{i} \in \mathcal{D}_{y}$. As in Section 3.1, we consider the case where health data are modelled as (conditionally) independent Poisson variables, so that

$$
Y_{k}\left(\mathbf{s}_{i}\right) \mid \eta_{k}\left(\mathbf{s}_{i}\right) \stackrel{i n d}{\sim} \operatorname{Po}\left(E_{k}\left(\mathbf{s}_{i}\right) e^{\eta_{k}\left(\mathbf{s}_{i}\right)}\right)
$$

with $\eta_{k}\left(\mathbf{s}_{i}\right)$ being the $\log$ relative risk in areal unit $\mathbf{s}_{i}$ for disease $k$. The linear predictor can be specified as

$$
\eta_{k}\left(\mathbf{s}_{i}\right)=\mu_{k}\left(\mathbf{s}_{i}\right)+\phi_{k}\left(\mathbf{s}_{i}\right)
$$

where $\mu_{k}\left(\mathbf{s}_{i}\right)$ and $\phi_{k}\left(\mathbf{s}_{i}\right)$ represent the mean level component and the spatial random effect at area $\mathbf{s}_{i}$ for disease $k$, respectively. In matrix form, the linear predictor in equation (2) can be written as

$$
\eta\left(\mathbf{s}_{i}\right)=\mu\left(\mathbf{s}_{i}\right)+\phi\left(s_{i}\right),
$$

where $\boldsymbol{\mu}\left(\mathbf{s}_{i}\right)$ and $\phi\left(s_{i}\right)$ are $n_{y}$-dimensional vectors of fixed and random effects, respectively, for the $n_{y}$ diseases at site $s_{i}$. In general, multivariate models differ mainly with respect to the specification of the joint distribution of the random effects and, more specifically, on how dependences within and between diseases are defined. Henceforth, we shall discuss solutions which either generalise CAR models to a multivariate framework or follow a factor-analytic approach.

\subsubsection{Multivariate CAR models}

Univariate CAR models are defined by the conditional distribution of the variable at each site given the variable at all the other sites. The extension to the multivariate case follows the same rationale. The more usual generalisation (Mardia, 1988), considers the $N_{y}$ Gaussian conditional distributions $\phi\left(s_{i}\right) \mid \phi\left(-s_{i}\right)$ of the $n_{y}$-vector variable $\phi\left(s_{i}\right)$ at each 
site $\mathbf{s}_{i}$ given $\phi\left(-\boldsymbol{s}_{i}\right)$, where $\phi\left(-\boldsymbol{s}_{i}\right)$ denotes the values $\phi\left(\boldsymbol{s}_{j}\right)$ at all sites $\boldsymbol{s}_{j} \neq \boldsymbol{s}_{i}$. The conditional expectations and variances are specified as

$$
E\left(\phi\left(\mathbf{s}_{i}\right) \mid \phi\left(-\mathbf{s}_{i}\right)\right)=\sum_{j} \mathbf{A}_{i j} \phi\left(\mathbf{s}_{j}\right), \quad \operatorname{Var}\left(\phi\left(\mathbf{s}_{i}\right) \mid \phi\left(-\mathbf{s}_{i}\right)\right)=\boldsymbol{\Gamma}_{i},
$$

where $\mathbf{A}_{i j}$ are matrices of parameters relating to the cross-dependences of the variables and $\boldsymbol{\Gamma}_{i}$ is the $n_{y} \times n_{y}$ conditional covariance matrix. Again, as in the univariate case, the conditional dependence structure is limited to neighboring areas identified by the adjacency matrix $\mathbf{W}$ such that $\mathbf{A}_{i j} \neq \mathbf{0}$ if and only if $w_{i j} \neq 0$.

Let $\boldsymbol{\phi}_{s}=\left(\boldsymbol{\phi}\left(\mathbf{s}_{1}\right)^{\prime}, \ldots, \boldsymbol{\phi}\left(\mathbf{s}_{N_{y}}\right)^{\prime}\right)^{\prime}$ denote the $N_{y} n_{y}$-vector of random effects ordered by site. Also, let us define $\boldsymbol{\phi}_{v}=\left(\boldsymbol{\phi}_{1}\left(\mathbf{s}_{1}, \ldots, \mathbf{s}_{N_{v}}\right)^{\prime}, \ldots, \boldsymbol{\phi}_{n_{v}}\left(\mathbf{s}_{1}, \ldots, \mathbf{s}_{N_{v}}\right)^{\prime}\right)^{\prime}$ as the same vector with random effects ordered by variables, which shows clearly the relevant contribution of each disease and the joint contributions of pairs of diseases. Clearly, the two forms are just different ways of representing the same multivariate process. One form may sometimes be more convenient to specify than the other. Under Gaussianity, given the $N_{y}$ conditional distributions, the joint distribution of $\boldsymbol{\phi}_{s}$ is multivariate Gaussian with mean zero and precision matrix $\mathbf{P}_{s}=\operatorname{Block}\left(-\boldsymbol{\Gamma}_{i}^{-1} \mathbf{A}_{i j}\right)$; the precision matrix $\boldsymbol{P}_{v}$ can be obtained by permuting the rows and the columns of $\mathbf{P}_{s}$. In order to satisfy the symmetry condition for the joint covariance matrix, the equality $\mathbf{A}_{i j} \boldsymbol{\Gamma}_{j}=\boldsymbol{\Gamma}_{i} \mathbf{A}_{j i}^{\prime}$ needs to hold. One difficulty concerning this approach is that conditions on the smoothing parameters for positive definiteness of the joint distribution precision matrix depend on the unknown conditional covariance matrix $\boldsymbol{\Gamma}$. A general strategy for checking positive definiteness of the precision matrices, as derived below, is proposed in Ippoliti et al. (2015).

Assumptions are needed to reduce the total number of parameters. For example, it is often reasonable to assume that the conditional variance $\boldsymbol{\Gamma}_{i}$ is either constant, or only varies over sites by a constant, so that $\boldsymbol{\Gamma}_{i}=w_{i+}^{-1} \boldsymbol{\Gamma}$. The multivariate generalisations of univariate CAR models proposed in the literature, mainly differ in the way in which both the spatial correlation and the cross-correlations are managed. A popular and fairly general approach is proposed by Jin et al. (2007). The approach is based on the linear model of coregionalization and encompasses several other models as special cases. The specification of the model begins with the definition of latent spatial effects, $\omega_{v}=$ $\left(\omega_{1}^{\prime}, \ldots, \omega_{k}^{\prime}, \ldots, \omega_{n_{y}}^{\prime}\right)^{\prime}$, where $\omega_{k}=\left(\omega_{k}\left(\mathbf{s}_{1}\right), \ldots, \omega_{k}\left(\mathbf{s}_{N_{y}}\right)\right), k=1, \ldots, n_{y}$, is a $N_{y}$-dimensional spatial process. The spatial effects for each disease are then modelled as $\boldsymbol{\phi}_{v}=\left(\mathbf{G} \otimes \mathbf{I}_{N_{y}}\right) \boldsymbol{\omega}_{v}$, where $\mathbf{G}$ is the upper triangular Cholesky decomposition of $\boldsymbol{\Gamma}$. A versatile model is obtained by assuming that the $\boldsymbol{\omega}_{k}$ are dependent and not identical latent processes so that the joint distribution of $\omega_{v}$ is $\operatorname{MVN}\left(\mathbf{0},\left(\mathbf{I}_{n_{y}} \otimes \mathbf{D}-\mathbf{B} \otimes \mathbf{W}\right)^{-1}\right)$, where $\mathbf{B}$ is a $n_{y}$-dimensional symmetric matrix whose diagonal entries capture the spatial autocorrelation for the $k$-th disease, and off-diagonal entries capture the spatial cross-correlation between diseases $k$ and $k^{\prime}$. This model is dubbed $\operatorname{MCAR}(\mathbf{B}, \boldsymbol{\Gamma})$ in what follows. It turns out that the joint distribution of the vector $\boldsymbol{\phi}_{\nu}$ is a zero mean multivariate Gaussian with precision matrix

$$
\mathbf{P}_{v}=\left(\mathbf{G} \otimes \mathbf{I}_{N_{y}}\right)\left(\mathbf{I}_{n_{y}} \otimes \mathbf{D}-\mathbf{B} \otimes \mathbf{W}\right)^{-1}\left(\mathbf{G} \otimes \mathbf{I}_{N_{y}}\right)^{\prime} .
$$

Conditions for positive definiteness of the joint precision matrix are obtained by constraining the eigenvalues of matrix $\mathbf{B}$ to lie between the reciprocal of the minimum and maximum eigenvalues of $\mathbf{D}^{-1 / 2} \mathbf{W D}^{-1 / 2}$. The $M C A R(\mathbf{B}, \boldsymbol{\Gamma})$ model belongs to the class of multivariate CAR models that explicitly model symmetric cross-covariances, and allows different disease-specific smoothing parameters.

A less general model (Carlin and Banerjee, 2003), which turns out to be a special case of the $\operatorname{MCAR}(\mathbf{B}, \boldsymbol{\Gamma})$ model, assumes one smoothing parameter for each disease and can be obtained by setting $\mathbf{B}=\operatorname{diag}\left(\rho_{1}, \ldots, \rho_{n_{y}}\right)$, delivering the $\operatorname{MCAR}\left(\rho_{1}, \ldots, \rho_{n_{y}}, \boldsymbol{\Gamma}\right)$ model. Conditions for positive definiteness require that $\left|\rho_{k}\right|<1, k=1, \ldots, n_{y}$.

The MCAR model proposed in Gelfand and Vounatsou (2003) assumes a common spatial smoothing parameter $\rho$ capturing disease-specific correlation and cross-correlations, such that the joint precision matrix can be obtained by setting $\mathbf{B}=\rho \mathbf{I}_{n_{y}}$, delivering the $\operatorname{MCAR}(\rho, \boldsymbol{\Gamma})$. According to this specification, the condition for positive definiteness of the covariance matrix reduces to $|\rho|<1$ as in the univariate framework. This approach delivers an immediate and intuitive generalisation to the multivariate framework with the evident drawback that a single smoothing parameter $\rho$ is employed to capture disease-specific spatial association and cross covariances. Note that the multivariate intrinsic CAR distribution is obtained if $\rho=1$.

The $\operatorname{MCAR}(\rho, \boldsymbol{\Gamma}), \operatorname{MCAR}\left(\rho_{1}, \ldots, \rho_{n_{y}}, \boldsymbol{\Gamma}\right)$ and $\operatorname{MCAR}(\mathbf{B}, \boldsymbol{\Gamma})$ models improve each other with respect to the generality of the spatial correlation and cross-correlation structure considered, the latter model being the most general one, with the only unsatisfactory restriction of specifying symmetric cross-covariances. A first generalisation of the CAR distribution to a multivariate framework allowing non-symmetric cross-covariances was proposed by Kim et al. 
(2001), by specifying univariate full conditionals; however the extension of the model to the case where $n_{y}>2$ appears unfeasible.

In Jin et al. (2005) an approach based on the direct specification of the joint distribution for the multivariate spatial process through the specification of marginal and conditional distributions is proposed. Although this approach allows for the specification of asymmetric covariance structures, it has the main drawback that the conditioning order has an effect on the results and it is difficult to apply when $n_{y}$ is large.

A more general $\operatorname{MCAR}(\tilde{\mathbf{B}}, \boldsymbol{\Gamma})$ asymmetric model, suited for the case where $n_{y}>2$, has been proposed by Greco and Trivisano (2009). As for the $\operatorname{MCAR}(\mathbf{B}, \mathbf{\Gamma})$ model, the model of Greco and Trivisano (2009) starts from the linear model of coregionalisation but allows the off-diagonal elements of $\mathbf{B}$ to be different, obtaining the joint covariance matrix as

$$
\boldsymbol{P}_{v}=\left(\mathbf{G} \otimes \mathbf{I}_{N_{y}}\right)\left(\mathbf{I}_{n_{y}} \otimes \mathbf{D}-\left(\tilde{\mathbf{B}} \otimes \mathbf{W}_{U}+\tilde{\mathbf{B}}^{\prime} \otimes \mathbf{W}_{U}^{\prime}\right)\right)^{-1}\left(\mathbf{G} \otimes \boldsymbol{I}_{N_{y}}\right)^{\prime}
$$

where $\mathbf{W}_{U}$ denotes the lower triangular part of the adjacency matrix $\mathbf{W}$ and $\tilde{\mathbf{B}}_{k k^{\prime}} \neq \tilde{\mathbf{B}}_{k^{\prime} k}$. Conditions for positive definiteness of the joint covariance matrix are obtained by constraining the singular values of $\tilde{\mathbf{B}}$ to lie between 0 and 1. The $\operatorname{MCAR}(\mathbf{B}, \boldsymbol{\Gamma})$ model arises as a special case of this model when $\tilde{\mathbf{B}}$ is constrained to be symmetric.

The model proposed by Sain and Cressie (2007), dubbed by the authors as the canonical multivariate conditional autoregressive model (CAMCAR), is characterized by a very similar specification with more restrictive conditions for positive definiteness with respect to those found in Greco and Trivisano (2009). Sain et al. (2011) also provide a different representation of multivariate lattice data that was suggested, but not implemented, in Sain and Cressie (2007). The idea is to think of the multivariate process as a univariate CAR over $N_{y} n_{y}$ regions, so that the process can be expressed through the individual conditional distributions, $\phi_{k}\left(\mathbf{s}_{i}\right) \mid \phi_{-k}\left(-\mathbf{s}_{i}\right)$, of each variable at each site. This formulation of a multivariate MRF allows great flexibility in modelling the conditional dependence structure and is easily extendable to complex neighborhood structures.

It is also worth mentioning that a partial review on GMRF/CAR and multivariate GMRF prior formulations, both for univariate and multivariate disease mapping models, is presented in MacNab (2011) giving insights into various prior characteristics for representing disease risks variability and spatial interaction.

Finally we note that, together with multivariate CARs, purely factor models (see Section 3.2.2) and smoothed analysis of variance (Zhang et al., 2009) also represent an alternative approach for the analysis of multiple diseases. Martinez-Beneito (2013) describes a general modelling approach that combines several different spatial structures with different multivariate dependence schemes. An alternative reformulation that accrues substantial computational benefits enabling the joint mapping of several diseases is also discussed by Botella-Rocamora et al. (2015).

\subsubsection{Latent variable models}

Multivariate spatial analysis becomes computationally onerous as soon as the number of geographical units is large and more than two or three diseases are available - see, for example, Dobra et al. (2011). Therefore, the development of computationally efficient multivariate models is desirable.

One main question, both from a methodological and an applied standpoint, is how to condense the available information into interpretable aggregates. One possibility is to use Factor Analysis (FA), which is a dimension reduction method to search for the underlying structures of multiple diseases. In practice, it is assumed that diseases that are from the same population groups or areas, often exhibit similar characteristics leading to the belief that they might be driven by some common sources, often referred to as common factors.

The use of a factor-analytic approach enjoys several advantages. For example, with the identification of a (usually small) number of latent variables, FA avoids the curse of dimensionality commonly present in large spatial data. Also, since the variables are often correlated with each other and each of the variables might also be correlated across the locations due to geographic similarities, FA facilitates the identification of spatial clusters in the latent factors which further avoids dimensionality issues. Finally, for studies considering the combined effects of multiple pollutants simultaneously, there might be the need of constructing appropriate air quality indicators based on dimensionality reduction techniques (Rushworth et al., 2014). In this case, FA enables all available pollutants to be combined and used to estimate the health impact of a proxy measure of the air pollution in ecological-type regression.

A spatial factor model can be specified through the linear predictor

$$
\eta_{k}\left(\mathbf{s}_{i}\right)=\mu_{k}\left(\mathbf{s}_{i}\right)+\sum_{j=1}^{m} \lambda_{k j} f_{y, j}\left(\mathbf{s}_{i}\right), \quad k=1, \ldots, n_{y}
$$


in which, compared with equation (2), the random effect $\phi_{k}\left(\mathbf{s}_{i}\right)$ is now rewritten as a truncated expansion in which $\left\{f_{y, j}\left(\mathbf{s}_{i}\right) \mid j=1, \ldots, m\right\}$ are $m$ common spatial factor processes underlying the $n_{y}$ observed diseases and $\lambda_{k j}$ is the factor loading for variable (disease) $k$ on the $j$-th factor. The factor $\mathbf{f}_{y}\left(\mathbf{s}_{i}\right)=\left(f_{y, 1}\left(\mathbf{s}_{i}\right), \ldots, f_{y, m}\left(\mathbf{s}_{i}\right)\right)^{\prime}$ is assumed to be an $m$-dimensional stationary process, with $m \ll n_{y}$, such that $E\left(\mathbf{f}_{y}(\mathbf{s})\right)=\boldsymbol{\mu}_{f}$ and $\operatorname{Cov}\left(\mathbf{f}_{y}(\mathbf{s})\right)=\boldsymbol{\Sigma}_{f}$.

In matrix form, the factor model in equation (4) can be written as

$$
\boldsymbol{\eta}\left(\mathbf{s}_{i}\right)=\boldsymbol{\mu}\left(\mathbf{s}_{i}\right)+\boldsymbol{\Lambda} \mathbf{f}_{y}\left(\mathbf{s}_{i}\right),
$$

where $\boldsymbol{\eta}\left(\mathbf{s}_{i}\right)=\left(\eta_{1}\left(\mathbf{s}_{i}\right), \ldots, \eta_{n_{y}}\left(\mathbf{s}_{i}\right)\right)^{\prime}$ and $\boldsymbol{\Lambda}=\left[\lambda_{k j}\right]$ is a $n_{y} \times m$ factor-loading matrix.

Despite its simplicity, this model poses several important statistical issues. For example, though cross-loadings are in general allowed to be estimated in $\boldsymbol{\Lambda}$, it is common practice to assume that the $m$ underlying factors are related only to their own manifest variables. Models with this simple structure are transparent and easily interpreted and have also been suggested by Liu et al. (2005). Another possibility is to induce sparsity in the loading matrix. This is especially useful in cases where $n_{y}$ is large. Moreover, this choice naturally helps to overcome the drawbacks of FA, such as unidentifiability with respect to the rotation of the latent matrices, and the difficulty of selecting the appropriate number of factors. In fact, by imposing substantial regularization on $\boldsymbol{\Lambda}$, the identifiability issue can be alleviated when the latent space is sufficiently sparse, and model selection criteria appears to be more effective at choosing the number of factors because the model does not overfit to the same extent as a non-sparse model. There are currently a number of options for how to induce sparsity constraints on the latent parameter space.

So called zero-norm priors assign finite probability mass to sparse solutions and MCMC techniques are typically used to solve the resulting intractable inference problem (Mitchell and Beauchamp, 1988; West, 2003; Carvalho et al., 2008). An alternative approach is to use the so called shrinkage priors which are continuous heavy-tailed densities which favour sparse solutions. The use of shrinkage priors is more closely related to non-Bayesian sparse estimation techniques. The canonical example is the Laplace distribution which leads to $L 1$ or LASSO regularisation under Maximum a Posteriori (MAP) parameter estimation (Tibshirani, 1996; Williams, 1995). LASSO regularisation has been used for the closely related problem of sparse principal component analysis (see, for example, Zou et al., 2006).

Shrinkage priors offer considerable computational advantages over zero-norm priors because they transform an inference problem over discrete parameters into a continuous problem which is more easily addressed using standard deterministic approximate inference methods (Seeger, 2008). However, although Maximum a Posteriori parameter estimates obtained with shrinkage priors are sparse, samples from the posterior distribution will not be truly sparse. This is a significant drawback if one is interested in characterising the uncertainty about whether or not a parameter is exactly zero.

In the framework of hierarchical Bayesian models, it would be preferable to focus on zero-norm priors which do assign finite probability mass to sparse solutions. These priors better characterise a prior belief in sparsity and should therefore lead to more meaningful posterior beliefs. A natural implementation of a zero-norm sparsity prior in this context is a spike and slab prior. This is a mixture prior on the entries of the factor loading matrix, where one mixture component drives the weight to zero while the other mixture component allows for non-zero entries. This prior, suggested by West (2003), not only assigns finite probability mass to truly sparse solutions, but also allows available information about the sparse structure to be included in a natural and interpretable manner: prior probabilities over specific entries in the mixing matrix can be used to adjust the relative weights of the corresponding mixture components.

A further issue is that $\mathbf{f}_{y}(\mathbf{s})$ also requires the specification of flexible multivariate covariance structures since we should model not only between-variable covariance but also across-location covariance. To this end, assuming a Gaussian prior and following Liu et al. (2005), it can be shown that a rich and flexible class of variance-covariance structures can be specified by using the linear model of coregionalization as discussed, for example, in Schmidt and Gelfand (2003) and Banerjee et al. (2004).

Finally, we note that performing a dimensionality reduction procedure on exposure variables, as specified in equation (4), and then regressing $\mathbf{f}_{y}(\mathbf{s})$ onto $\mathbf{f}_{x}(\mathbf{s})$ in a second level of the hierarchy, offers a possibility to overcome the problem of multicollinearity for a set of exposure variables. As shown in Liu et al. (2005), Fontanella et al. (2015), Ippoliti et al. (2012) and Valentini et al. (2013), this approach allows for a fully Bayesian specification of a Generalised Structural Equation model (GSEM). In this context, a confirmatory approach, in which each variable loads only onto a specific common factor, may facilitate the interpretation of latent variables as well as the effects of the exposure variables on the health variables. 


\subsection{Spatio-temporal regression}

As a result of the increasing availability of both air pollution and health data, ecological spatio-temporal studies have also been developed in literature. The hierarchical models relating air pollution and health discussed above, can be naturally extended to a space-time setting by using a linear function of air pollutants, covariates and space-time random effects. With the conventional assumption that $Y\left(\mathbf{s}_{i}, t\right)$ has a Poisson distribution, i.e.

$$
Y\left(\mathbf{s}_{i}, t\right) \mid \eta\left(\mathbf{s}_{i}, t\right) \stackrel{i n d}{\sim} \operatorname{Po}\left(E\left(\mathbf{s}_{i}, t\right) e^{\eta\left(\mathbf{s}_{i}, t\right)}\right),
$$

a quite general model specifies the linear predictor as

$$
\eta\left(\mathbf{s}_{i}, t\right)=\mu\left(\mathbf{s}_{i}, t\right)+\phi\left(\mathbf{s}_{i}, t\right)
$$

where $\mu\left(\mathbf{s}_{i}, t\right)$ represents the large-scale spatio-temporal variability of the log-relative risk, which of course can be modelled as a function of exposure variables at time $t$ and area $\boldsymbol{s}_{i}$ as $\mu\left(\mathbf{s}_{i}, t\right)=\tilde{\boldsymbol{x}}\left(\mathbf{s}_{i}, t\right)^{\prime} \boldsymbol{\beta}$. Random effects $\phi\left(\mathbf{s}_{i}, t\right)$ are introduced to capture any residual spatio-temporal autocorrelation. A commonly used approach has been proposed by Knorr-Held (2000) where a set of independent random components explains the overall risk effect over the space-time domain using

$$
\phi\left(\mathbf{s}_{i}, t\right)=\psi\left(\mathbf{s}_{i}\right)+v\left(\mathbf{s}_{i}\right)+\delta(t)+\zeta(t)+\theta\left(\mathbf{s}_{i}, t\right)
$$

where $v\left(\mathbf{s}_{i}\right)$ and $\zeta(t)$ are independent zero mean Gaussian random effects with common variance (i.e. the heterogeneity components) and $\psi\left(\mathbf{s}_{i}\right)$ and $\delta(t)$ are spatial and temporal random effects represented by the intrinsic CAR and first order random walk priors, respectively. The space-time random effect $\theta\left(\mathbf{s}_{i}, t\right)$ has a Gaussian prior with general covariance matrix representing cases of independence, spatial, temporal and/or spatio-temporal autocorrelation (Knorr-Held, 2000).

Another popular approach is to allow the spatially smoothed log-risk surface to evolve over time via an autoregressive process. Denoting with $\boldsymbol{\phi}_{t}=\left(\phi\left(\mathbf{s}_{1}, t\right), \ldots, \phi\left(\mathbf{s}_{N_{y}}, t\right)\right)^{\prime}$, it may be assumed, for example, that $p\left(\boldsymbol{\phi}_{1}, \ldots, \boldsymbol{\phi}_{T}\right)=$ $p\left(\phi_{1}\right) \prod_{t=2}^{T} p\left(\phi_{t} \mid \phi_{t-1}\right)$ for which, under Gaussian priors, the marginal distribution of $\phi_{1}$ has mean zero and covariance matrix $\boldsymbol{P}^{-1}$ while the conditional distributions, $\boldsymbol{\phi}_{t} \mid \boldsymbol{\phi}_{t-1}$, have conditional means which evolve in time according to a first order autoregressive process. The matrix $\boldsymbol{P}$ is a precision matrix usually specified through a CAR. This specification is useful to model the long-term effects of air pollution on health and has been used by Ugarte et al. (2012) and Rushworth et al. (2014). Note that an extension of this model specification to higher order autoregressive processes or, alternatively, the use of a Space Time Autoregressive Generalised (STARG) prior (Di Giacinto et al., 2005) is in general possible. However, their use is subject to the availability of spatial series which are rich in time.

Other papers dealing with spatio-temporal data include Elliott et al. (2007), Janes et al. (2007), Choi et al. (2009), Lawson et al. (2010), Choi et al. (2011), Greven et al. (2011) and Lawson et al. (2012), with the latter being the only paper considering confounding bias problems in space-time models.

Extending the space-time models above to a multivariate setting is not straightforward. On the other hand, Bayesian factor models are common in multivariate time series analysis (Aguilar and West, 2000; Lopes and West, 2004) and, in this framework, the multivariate spatial structure of the geographical units suggests an extension of the usual dynamic factor models to make use of the similarity between adjacent regions. However, the literature of spatial dynamic factor models in health care research, especially for short-term effect studies, is still sparse and most of the applications discuss the use of FA to achieve a dimensionality reduction of a set of covariates. An example is offered by Reich et al. (2008) who extend the usual dynamic factor model by borrowing strength across neighboring diameters of particles and then using the latent factors as predictors of mortality.

A new class of spatial dynamic factor models for measurements belonging to the exponential family of distributions is also discussed by Lopes et al. (2011). Though the model was developed only for one dependent variable, it represents a direct extension to a space-time setting of the spatial factor model of equation (4). In their formulation, the factor loadings matrix is responsible for modelling spatial variation, while the common factors capture the temporal variation. In the development of a Generalized Factor model, we use the link function to relate the conditional mean to the linear predictor. However, in general, this does not require $\eta_{k}(\cdot)$ to be linear in $f_{y, j}(\cdot)$. In particular, one could think of $\eta_{k}(\cdot)$ as an additive function of $f_{y, j}(\cdot)$ in the form of a generalized additive model (GAM, Hastie and Tibshirani (1990)). 
Assuming normality, a more structured version of the model proposed by Lopes et al. (2011) can be found in Ippoliti et al. (2012) and Valentini et al. (2013), with the latter discussing a multivariate extension - i.e. $n_{y}>1$. Although this extension was not proposed in the framework of health care research, it can be a valuable tool in this framework, offering simple solutions to the spatial misalignment problem.

\section{Available software}

Bayesian inference for the spatial and spatio-temporal models described in Section 3 is carried out using MCMC methods (Robert and Casella, 2004; Brooks et al., 2011). A way for obtaining MCMC inference consists in running MCMC simulations by means of hand writing code, a solution which is flexible and tailored to the specific application but is obviously the most error-prone. Alternatively, it is possible to use a software environment designed to allow users to perform Bayesian inference via MCMC. For example, the BUGS (Bayesian inference Using Gibbs Sampling) project (http://www.mrc-bsu.cam.ac.uk/software/bugs/) has given rise to the WinBUGS (Lunn et al., 2000, 2012) software, which has opened the doors of Bayesian modelling to the wide research community. Moreover, starting from 2004 there exists also an open-source version of the core BUGS code named OpenBUGS (http: //www . openbugs . net/); see Lunn et al. (2009) for a history of the BUGS project. Note that WinBUGS and OpenBUGS can also be run within other programs like R (through the R2OpenBUGS, R2WinBUGS and BRugs packages), Stata and SAS. Another possibility is the R CARBayes package developed by Duncan Lee (Lee, 2013) for the implementation of Bayesian hierarchical spatial areal unit models, characterized by random effects with conditional autoregressive prior distribution. In general the $\mathrm{R}$ community is extremely active in the development of new packages, listed in the CRAN task view for Bayesian inference (https://cran.r-project.org/web/views/Bayesian.html) and analysis of spatial data (https://cran.r-project.org/web/views/Spatial.html).

Recently, an alternative to MCMC has been proposed by Rue et al. (2009) based on the Integrated Nested Laplace Approximation (INLA) approach, which is designed for latent Gaussian models, a wide and flexible class of models ranging from (generalized) linear mixed to spatial and spatio-temporal models. INLA, which can be run through the R-INLA package (http://www.r-inla.org/), is a deterministic algorithm for Bayesian inference and provides accurate results in short computing time. For this reason it can be preferred when model complexity and database dimension are troublesome from a computational point of view. Its use is now well established in several research fields, including ecology, epidemiology, econometrics and environmental science (Rue et al., 2016); for more details about INLA for spatial and spatio-temporal models we refer the reader to Blangiardo et al. (2013) and Blangiardo and Cameletti (2015), where practical examples and code guidelines are also provided. Moreover, see the recent papers Gerber and Furrer (2015) and Carroll et al. (2015) for an up-to-date comparison between INLA and MCMC methods.

\section{Discussion}

This paper has reviewed the statistical challenges involved in estimating the health impact of air pollution using spatial and spatio-temporal ecological regression, typically based on Poisson log-linear models. An important branch of the literature which has not been covered in this review concerns point process models to analyse case event data, i.e. geo-referenced case occurrences (Diggle, 2003; Lawson, 2012). On the one hand, case event data are appealing since they preserve exact spatial information concerning the disease occurrences, even though sometimes the use of residential address is poorly related to individual exposure. A relevant example of point process methods in spatial epidemiology is when case-control matched data are used to investigate the health status of people living around potential environmental pollution sources, such as an incinerator (Diggle, 1990; Diggle et al., 2000). Controls are matched to cases to account for heterogeneity of the underlying population at risk. In this framework, point process methods are applied to estimate and compare the intensity surface of the point processes generating both controls and cases, in order to understand changes in relation to the location of the putative source of hazard. In general, different classes of point process models have been proposed in order to take into account general features of the data generating process: the most general class for taking account of smooth spatially varying intensity is that of Log-Gaussian Cox Processes (see Diggle (2014) for a comprehensive discussion) which allow inclusion of spatial random effects and covariate effects. This class of models have been recently applied in epidemiological studies by Pinto Junior et al. (2015) and Liang et al. (2008). 
Despite the problems associated with ecological studies (as discussed in Section 2), they are far more diffused in spatial epidemiology than studies based on case event data, not only because of their ready availability. In fact, count data are more closely matched with the background population at risk and exposure measurements are more reliable when they are expressed as average exposure at small area level than when they are expressed as individual level exposure (Lawson, 2006). Generally, the more refined the spatial scale of exposure, the higher the uncertainty associated with it; this can lead to biased estimates of the exposure-risk relationship. It should be noticed that almost ever the researcher cannot control data aggregation since count data are collected in administrative regions, as well as socio-demographic explanatory variables; hence ecological bias should be accounted for by means of statistical models. One possible approach to face problems arising in ecological regression studies consists in trying to estimate the joint distribution of response and explanatory variables within areas using a sample drawn from each area, using the collected information to adjust the ecological regression coefficients (Plummer and Clayton, 1996). However, this procedure is often not feasible in practice because of the high costs related to the sampling operations.

\section{Acknowledgements}

All the authors have been supported by the FIRB Project StEPhl (project no. RBFR12URQJ, http://stephiproject . it/) provided by the Italian Ministry for Education, University and Research.

\section{Bibliography}

\section{References}

Agarwal, D. K., Gelfand, A. E., Citron-Pousty, S., 2002. Zero-inflated models with application to spatial count data. Environmental and Ecological Statistics 9 (4), 341-355.

Aguilar, O., West, M., 2000. Bayesian dynamic factor models and portfolio allocation. Journal of Business and Economic Statistics $18,338-357$.

Atkinson, R. W., Fuller, G. W., Anderson, H. R., Harrison, R. M., Armstrong, B., 2010. Urban ambient particle metrics and health: A time-series analysis. Epidemiology 21 (4).

Atkinson, R. W., Mills, I. C., Walton, H. A., Anderson, H. R., 2014. Fine particle components and health - systematic review and meta-analysis of epidemiological time series studies of daily mortality and hospital admissions. Journal of Exposure Science and Environmental Epidemiology 25 (2), 208-214.

Banerjee, S., Carlin, B., Gelfand, A., 2004. Hierarchical Modeling and Analysis for Spatial Data. Monographs on Statistics and Applied Probability. Chapman and Hall, New York.

Basagaña, X., Aguilera, I., Rivera, M., Agis, D., Foraster, M., Marrugat, J., Elosua, R., Künzli, N., 2013. Measurement error in epidemiologic studies of air pollution based on land-use regression models. American Journal of Epidemiology 178 (8), $1342-1346$.

Bayarri, M., Berger, J., Steinberg, D., 2009. Special issue on computer modeling. Technometrics 51 (4), $353-353$.

Bell, M. L., 2006. The use of ambient air quality modeling to estimate individual and population exposure for human health research: A case study of ozone in the Northern Georgia Region of the United States. Environment International 32 (5), 586 - 593.

Berhane, K., Gauderman, W., Stram, D., Thomas, D., 2004. Statistical issues in studies of the long-term effects of air pollution: The Southern California Children's Health Study. Statistical Science 19 (3), 414-449.

Berrocal, V., Gelfand, A., Holland, D., 2011. Space-time data fusion under error in computer model output: An application to modeling air quality. Biometrics.

Besag, J., York, J., Mollié, A., 1991. Bayesian image restoration, with two applications in spatial statistics. Annals of the Institute of Statistical Mathematics 43 (1), 1-20.

Best, N., Richardson, S., Thomson, A., 2005. A comparison of Bayesian spatial models for disease mapping. Statistical Methods in Medical Research 14 (1), 35-59.

Biggeri, A., Bellini, P., Terracini, B., 2004. Metanalisi italiana degli studi sugli effetti a breve termine dell'inquinamento atmosferico $1996-2002$. Epidemiologia e Prevenzione (Special Issue) 28 (4-5), 1-100.

Blangiardo, M., Cameletti, M., 2015. Spatial and Spatio-temporal Bayesian Models with R-INLA. Wiley.

Blangiardo, M., Cameletti, M., Baio, G., Rue, H., 2013. Spatial and spatio-temporal models with R-INLA. Spatial and Spatio-temporal Epidemiology 4, $33-49$.

Blangiardo, M., Finazzi, F., Cameletti, M., 2016. Two-stage bayesian model to evaluate the effect of air pollution on chronic respiratory diseases using drug prescriptions. Spatial and Spatio-temporal Epidemiology, -Accepted - In press. URL \{http://dx.doi.org/10.1016/j.sste.2016.03.001\}

Blangiardo, M., Hansell, A., Richardson, S., 2011. A bayesian model of time activity data to investigate health effect of air pollution in time series studies. Atmospheric Environment 45, 379-386.

Botella-Rocamora, P., Martinez-Beneito, M., Banerjee, S., 2015. A unifying modeling framework for highly multivariate disease mapping. Statistics in Medicine 34 (9), 1548-1559.

Breslow, N., Day, N., 1980. Statistical methods in cancer research. Lyon, France, IARC Scientific Publications. 
Brook, R. D., Rajagopalan, S., Pope, C. A., Brook, J. R., Bhatnagar, A., Diez-Roux, A. V., Holguin, F., Hong, Y., Luepker, R. V., Mittleman, M. A., Peters, A., Siscovick, D., Smith, S. C., Whitsel, L., Kaufman, J. D., 2010. Particulate matter air pollution and cardiovascular disease: an update to the scientific statement from the American Heart Association. Circulation 121, 12331-2378.

Bo Brooks, S., Gelman, A., Jones, G., Meng, X. (Eds.), 2011. Handbook of Markov Chain Monte Carlo. CRC Press, Taylor \& Francis Group.

Brown, P., 1993. Measurement, Regression and Calibration. Oxford University Press.

Brunekreef, B., Holgate, S. T., 2002. Air pollution and health. The lancet 360 (9341), 1233-1242.

Bruno, F., Cocchi, D., Greco, F., Scardovi, E., 2014. Spatial reconstruction of rainfall fields from rain gauge and radar data. Stochastic Environmental Research and Risk Assessment 28 (5), 1235-1245.

Bruno, F., Paci, L., 2013. Spatio-temporal model for short-term predictions of air pollution data. In: Lanzarone, E., Ieva, F. (Eds.), The contribution of Young Researchers to Bayesian Statistics - Proceedings of BAYSM2013. Milan, 5-6 June, 2013.

Cameletti, M., Ignaccolo, R., Bande, S., 2011. Comparing spatio-temporal models for particulate matter in Piemonte. Environmetrics 22, 985-996.

Carey, I. M., Atkinson, R. W., Kent, A. J., van Staa, T., Cook, D. G., Anderson, H. R., 2013. Mortality associations with long-term exposure to outdoor air pollution in a national english cohort. American Journal of Respiratory and Critical Care Medicine 187 (11), $1226-1233$.

Carlin, B., Banerjee, S., 2003. Hierarchical multivariate car models for spatio-temporally correlated survival data (with discussion). In: Bernardo, J., Bayarri, M., Berger, J., Dawid, A., Heckerman, D., Smith, A., West, M. (Eds.), Bayesian Statistics 7. Oxford University Press, London, pp. 45-63.

Carlin, B., Xia, H., Devine, O., Tolbert, P., Mulholland, J., 1999. Spatio-Temporal Hierarchical Models for Analyzing Atlanta Pediatric Asthma ER Visit Rates. In: Gatsonis, C., Kass, R., Carlin, B., Carriquiry, A., Gelman, A., Verdinelli, I., West, M. (Eds.), Case Studies in Bayesian Statistics. Vol. 140 of Lecture Notes in Statistics. Springer New York, pp. 303-320.

Carpenter, T., 2011. The spatial epidemiologic (r)evolution: A look back in time and forward to the future. Spatial and Spatio-temporal Epidemiology 2 (3), 119 - 124, special Issue: \{GEOVET\} 2010.

Carroll, R., Lawson, A., Faes, C., Kirby, R., Aregay, M., Watjou, K., 2015. Comparing INLA and OpenBUGS for hierarchical Poisson modeling in disease mapping. Spatial and Spatio-temporal Epidemiology 14-15, $45-54$.

Carroll, R., Ruppert, D., Stefanski, L., Crainiceanu, C., 2006. Measurement Error in Nonlinear Models: A Modern Perspective, 2nd edn. Chapman and Hall/CRC: Boca Raton, FL.

Carvalho, C., Chang, J., Lucas, J., Nevins, J., Wang, Q., West, M., 2008. High-dimensional sparse factor modeling: Applications in gene expression genomics. Journal of the American Statistical Association 103 (484), 1438-1456.

Cesaroni, G., Badaloni, C., Gariazzo, C., Stafoggia, M., Sozzi, R., Davoli, M., Forastiere, F., 2013. Long-term exposure to urban air pollution and mortality in a cohort of more than a million adults in rome. Environmental Health Perspectives 121 (3), 324-331.

Choi, J., Fuentes, M., Reich, B. J., 2009. Spatial-temporal association between fine particulate matter and daily mortality. Computational Statistics \& Data Analysis 53 (8), $2989-3000$.

Choi, J., Lawson, A., Cai, B., Hossain, M., 2011. Evaluation of Bayesian spatial-temporal latent models in small area health data. Environmetrics 22 (8), 1008-1022.

Clayton, D., Bernardinelli, L., Montomoli, C., 1993. Spatial correlation in ecological analysis. International Journal of Epidemiology 22, 11931202.

Cocchi, D., Greco, F., Trivisano, C., 2007. Hierarchical space-time modelling of PM10 pollution. Atmospheric Environment 41 , 532-542.

Cressie, N., 1993. Statistics for Spatial Data. Wiley.

Di Giacinto, V., Dryden, I., Ippoliti, L., Romagnoli, L., 2005. Linear smoothing of noisy spatial temporal series. Journal of Mathematics and Statistics 1 (4), 300-312.

Diggle, P., 1990. A point process modelling approach to raised incidence of a rare phenomenon in the vicinity of a prespecified point. Journal of the Royal Statistical Society. Series A (Statistics in Society) 153 (3), 349-362.

Diggle, P., 2003. Statistical Analysis of Spatial Point Patterns. Mathematics in biology. Arnold.

Diggle, P., 2014. Statistical Analysis of Spatial and Spatio-Temporal Point Patterns, Third Edition. Chapman and Hall/CRC, New York.

Diggle, P. J., Morris, S. E., Wakefield, J. C., 2000. Point-source modelling using matched case-control data. Biostatistics 1 (1), 89-105. URL http://biostatistics.oxfordjournals.org/content/1/1/89. abstract

Dobra, A., Lenkoski, A., Rodriguez, A., 2011. Bayesian inference for general gaussian graphical models with application to multivariate lattice data. Journal of the American Statistical Association 106 (496), 1418-1433.

Dockery, D. W., Pope, C. A., Xu, X., Spengler, J. D., Ware, J. H., Fay, M. E., Ferris Jr, B. G., Speizer, F. E., 1993. An association between air pollution and mortality in six US cities. New England journal of medicine 329 (24), 1753-1759.

Eberly, L. E., Carlin, B. P., 2000. Identifiability and convergence issues for Markov chain Monte Carlo fitting of spatial models. Statistics in Medicine 19 (17-18), 2279-2294.

Elgethun, K., Fenske, R., Yost, M., Oalcisko, G., 2003. Time-location analysis for exposure assessment studies of children using a novel global positioning system instrument. Environ Health Perspect 111, 115-122.

Elliott, P., Shaddick, G., Wakefield, J. C., Hoogh, C. d., Briggs, D. J., 2007. Long-term associations of outdoor air pollution with mortality in Great Britain. Thorax 62 (12), 1088-1094.

Elliott, P., Wartenberg, D., 2004. Spatial epidemiology: Current approaches and future challenges. Environmental Health Perspectives 112 (9), 998-1006.

Evensen, G., 2009. Data assimilation. The Ensemble Kalman Filter. Springer.

85 Fassò, A., Cameletti, M., 2009. The EM algorithm in a distributed computing environment for modelling environmental space-time data. Environmental Modelling \& Software 24, 1027-1035.

Fassò, A., Cameletti, M., Nicolis, O., 2007. Air quality monitoring using heterogeneous networks. Environmetrics 18, $245-264$.

Fassò, A., Finazzi, F., 2011. Maximum likelihood estimation of the dynamic coregionalization model with heterotopic data. Environmetrics 22, 735-748.

Fontanella, L., Ippoliti, L., Sarra, A., Valentini, P., Palermi, S., 2015. Hierarchical generalised latent spatial quantile regression models with applications to indoor radon concentration. Stochastic Environmental Research and Risk Assessment 29 (2), $357-367$. 
Fuentes, M., Raftery, A., 2005. Model evaluation and spatial interpolation by Bayesian combination of observations with outputs from numerical models. Biometrics 61, 36-45.

794 Fuentes, M., Song, H.-R., Ghosh, S. K., Holland, D. M., Davis, J. M., 2006. Spatial association between speciated fine particles and mortality. Biometrics 62 (3), 855-863.

796 Fuller, W., 1987. Measurement Error Models. New York: John Wiley \& Sons.

797 Gelfand, A., 2012. Hierarchical modeling for spatial data problems. Spatial Statistics 1, $30-39$.

798 Gelfand, A., Diggle, P., Fuentes, M., Guttorp, P. (Eds.), 2010. Handbook of Spatial Statistics. Chapman \& Hall.

799 Gelfand, A., Vounatsou, P., 2003. Proper multivariate conditional autoregressive models for spatial data analysis. Biostatistics 4 (1), 11-15.

800 Gerber, F., Furrer, R., 2015. Pitfalls in the Implementation of Bayesian Hierarchical Modeling of Areal Count Data: An Illustration Using BYM and Leroux Models. Journal of Statistical Software 63 (1).

Gerharz, L., Krüger, A., Klemm, O., 2009. Applying indoor and outdoor modeling techniques to estimate individual exposure to PM2.5 from personal GPS profiles and diaries: a pilot study. Science of the Total Environment 407, 5184-5193.

Gotway, C., Young, L., 2002. Combining incompatible spatial data. Journal of the American Statistical Association 97 (458), $632-648$.

Gray, S., Gelfand, A., Miranda, M., 2011. Hierarchical spatial modeling of uncertainty in air pollution and birth weight study. Statistics in Medicine 30 (17), 2187-2198.

07 Greco, F. P., Trivisano, C., 2009. A multivariate CAR model for improving the estimation of relative risks. Statistics in Medicine 28 (12), 17071724.

09 Greenland, S., Morgenstern, H., 1989. Ecological bias, confounding and effects modification. International Journal of Epidemiology 18, $269-274$.

810 Greenland, S., Robins, J., 1994. Invited commentary: ecologic studies - biases, misconceptions, and counterexamples. American Journal of Epidemiology 139, 747-760.

Grell, K., Diggle, P., Frederiksen, K., Schüz, J., Cardis, E., Andersen, P., 2015. A three-dimensional point process model for the spatial distribution of disease occurrence in relation to an exposure source. Statistics in Medicine 34, 3170-3180.

Greven, S., Dominici, F., Zeger, S., 2011. An approach to the estimation of chronic air pollution effects using spatio-temporal information. Journal of the American Statistical Association 106 (494), 396-406.

Gryparis, A., Paciorek, C. J., Zeka, A., Schwartz, J., Coull, B. A., 2009. Measurement error caused by spatial misalignment in environmental epidemiology. Biostatistics 10 (2), 258-274.

Gschlößl, S., Czado, C., 2008. Modelling count data with overdispersion and spatial effects. Statistical Papers 49 (3), $531-552$.

819 Hastie, T., Tibshirani, R., 1990. Generalized Additive Models. Chapman \& Hall/CRC.

820 Hodges, J. S., Reich, B. J., 2010. Adding spatially-correlated errors can mess up the fixed effect you love. The American Statistician 64 (4), 325-334.

Hoek, G., Beelen, R., de Hoogh, K., Vienneau, D., Gulliver, J., Fischer, P., Briggs, D., 2008. A review of land-use regression models to assess spatial variation of outdoor air pollution. Atmospheric Environment 42 (33), $7561-7578$.

24 Hoek, G., Krishnan, R., Beelen, R., Peters, A., Ostro, B., Brunekreef, B., Kaufman, J., 2013. Long-term air pollution exposure and cardiorespiratory mortality: a review. Environmental Health 12 (1), 12-43.

Huang, G., Lee, D., Scott, M., 2015. An integrated Bayesian model for estimating the long-term health effects of air pollution by fusing modelled and measured pollution data: A case study of nitrogen dioxide concentrations in Scotland. Spatial and Spatio-temporal Epidemiology 14-15, 63 -74 .

Hubbell, B. J., Hallberg, A., McCubbin, D. R., Post, E., 2005. Health-related benefits of attaining the 8-hr ozone standard. Environmental Health Perspectives $113(1), 73-82$.

Hughes, J., Haran, M., 2013. Dimension reduction and alleviation of confounding for spatial generalized linear mixed models. Journal of the Royal Statistical Society: Series B (Statistical Methodology) 75 (1), 139-159.

Ignaccolo, R., Ghigo, S., Bande, S., 2013. Functional zoning for air quality. Environmental and Ecological Statistics 20, $109-127$.

Ignaccolo, R., Mateu, J., Giraldo, R., 2014. Kriging with external drift for functional data for air quality monitoring. Stochastic Environmental Research and Risk Assessment 28 (5), 1171-1186.

Ippoliti, L., Martin, R., L., R., 2015. A note on efficient likelihood computations for some multivariate Gaussian Markov Random Fields. Preprint available from the authors, Submitted.

38 Ippoliti, L., Valentini, P., Gamerman, D., 2012. Space-time modelling of coupled spatio-temporal environmental variables. Journal of the Royal Statistical Society, Series C 61, 175-200.

40 Jackson, C., Best, N., Richardson, S., 2008. Hierarchical related regression for combining aggregate and individual data in studies of socioeconomic disease risk factors. Statistics in Medicine 171.

42 Janes, H., Dominici, F., Zeger, S., 2007. Trends in air pollution and mortality: an approach to the assessment of unmeasured confounding. Epidemiology 18 (4), 416-23.

44 Jerrett, M., Gale, S., Kontgis, C., 2010. Spatial Modeling in Environmental and Public Health Research. International Journal of Environmental Research and Public Health 7 (4), 1302-1329.

Jin, X., Banerjee, S., Carlin, B. P., 2007. Order-free co-regionalized areal data models with application to multiple-disease mapping. Journal of the Royal Statistical Society: Series B (Statistical Methodology) 69 (5), 817-838.

848 Jin, X., Carlin, B. P., Banerjee, S., 2005. Generalized hierarchical multivariate CAR models for areal data. Biometrics 61 (4), $950-961$.

849 Kelsall, J., Wakefield, J., 2002. Modeling spatial variation in disease risk. Journal of the American Statistical Association 97 (459), 692-701.

850 Kim, H., Sun, D., Tsutakawa, R., 2001. A bivariate Bayes method for improving the estimates of mortality rates with a twofold conditional autoregressive model. Journal of the American Statistical Association 96, 1506-1521.

852 Knorr-Held, L., 2000. Bayesian modelling of inseparable space-time variation in disease risk. Environmetrics 19 (17), $2555-67$.

853 Krall, J. R., Chang, H. H., Sarnat, S. E., Peng, R. D., Waller, L. A., 2015. Current methods and challenges for epidemiological studies of the associations between chemical constituents of particulate matter and health. Current Environmental Health Reports 2 (4), $388-398$.

855 Künzli, N., 2012. Is air pollution of the 20th century a cause of current asthma hospitalisations? Thorax 67 (1), 2-3.

856 Lanki, T., Pekkanen, J., Aalto, P., Elosua, R., Berglind, N., D’Ippoliti, D., Kulmala, M., Nyberg, F., Peters, A., Picciotto, S., Salomaa, V., Sunyer, 
J., Tiittanen, P., von Klot, S., Forastiere, F., 2006. Associations of traffic related air pollutants with hospitalisation for first acute myocardial infarction: the HEAPSS study. Occupational and environmental medicine 63 (12), 844-851.

859 Lawson, A., 2006. Statistical Methods in Spatial Epidemiology. Wiley.

860 Lawson, A., 2009. Bayesian Disease Mapping. Hierarchical Modeling in Spatial Epidemiology. CRC Press.

861 Lawson, A., Choi, J., Cai, B., Hossain, M., Kirby, R., Liu, J., 2012. Bayesian 2-Stage Space-Time Mixture Modeling With Spatial Misalignment of the Exposure in Small Area Health Data. Journal of Agricultural, Biological, and Environmental Statistics 17 (3), $417-441$.

63 Lawson, A., Song, H., Cai, B., Hossain, M., Huang, K., 2010. Space-time latent component modeling of geo-referenced health data. Statistics in Medicine 29 (17), 2012-2027.

65 Lawson, A. B., 2012. Bayesian point event modeling in spatial and environmental epidemiology. Statistical Methods in Medical Research 21 (5), 509-529.

URL http://smm.sagepub.com/content/21/5/509.abstract

Lee, A., Szpiro, A., Kim, S., Sheppard, L., 2015. Impact of preferential sampling on exposure prediction and health effect inference in the context of air pollution epidemiology. Environmetrics 26 (4), 255-267.

70 Lee, D., 2013. CARBayes: An R package for Bayesian spatial modeling with conditional autoregressive priors. Journal of Statistical Software 55 (13), 1-24.

Lee, D., Rushworth, A., Sahu, S. K., 2014. A Bayesian localized conditional autoregressive model for estimating the health effects of air pollution. Biometrics 70 (2), 419-429.

74 Lee, D., Sarran, C., 2015. Controlling for unmeasured confounding and spatial misalignment in long-term air pollution and health studies. Environmetrics $26(7), 477-487$.

876 Lee, D., Shaddick, G., 2010. Spatial modeling of air pollution in studies of its short-term health effects. Biometrics 66 (4), $1238-1246$.

877 Lee, S.-J., Yeatts, K. B., Serre, M. L., 2009. A Bayesian Maximum Entropy approach to address the change of support problem in the spatial analysis of childhood asthma prevalence across North Carolina. Spatial and Spatio-temporal Epidemiology 1 (1), 49 - 60.

Lepeule, J., Laden, F., Dockery, D., Schwartz, J., 2012. Chronic exposure to fine particles and mortality: An extended follow-up of the harvard six cities study from 1974 to 2009 . Environmental Health Perspectives 120 (7), 965-970.

Li, L., Losser, T., Yorke, C., Piltner, R., 2014. Fast inverse distance weighting-based spatiotemporal interpolation: A web-based application of interpolating daily fine particulate matter PM2.5 in the contiguous U.S. using parallel programming and k-d tree. International Journal of Environmental Research and Public Health 11 (9), 9101.

84 Liang, S., Carlin, B., Gelfand, A., 2008. Analysis of Minnesota Colon and Rectum cancer point patterns with spatial and nonspatial covariate information. The annals of applied statistics 3 (3), 943-962.

Liu, X., Wall, M. M., Hodges, J. S., 2005. Generalized spatial structural equation models. Biostatistics 6 (4), $539-557$.

87 Lopes, H., Gamerman, D., Salazar, E., 2011. Generalized spatial dynamic factor models. Computational Statistics and Data Analysis 55, 13191330.

Lopes, H., West, M., 2004. Bayesian model assessment in factor analysis. Statistica Sinica 14, 41-67.

890 Lunn, D., Jackson, C., Best, N., Thomas, A., Spiegelhalter, D., 2012. The BUGS Book: A Practical Introduction to Bayesian Analysis. CRC Press. Lunn, D., Spiegelhalter, D., Thomas, A., Best, N., 2009. The BUGS project: Evolution, critique and future directions. Statistics in Medicine 25 (28), 3049-3067.

93 Lunn, D., Thomas, A., Best, N., Spiegelhalter, D., 2000. WinBUGS - A Bayesian modelling framework: Concepts, structure, and extensibility. Statistics and Computing 10 (4), 325-337.

95 MacNab, Y. C., 2003. Hierarchical bayesian modeling of spatially correlated health service outcome and utilization rates. Biometrics 59 (2), 305-315.

97 MacNab, Y. C., 2011. On Gaussian Markov random fields and Bayesian disease mapping. Statistical Methods in Medical Research 20 (1), 49-68.

98 Madsen, L., Ruppert, D., Altman, N. S., 2008. Regression with spatially misaligned data. Environmetrics 19 (5), $453-467$.

899 Mardia, K., 1988. Multi-dimensional multivariate gaussian markov random fields with applications to image processing. Journal of Multivariate Analysis 24, 265-284.

1 Martinez-Beneito, M., 2013. A general modelling framework for multivariate disease mapping. Biometrika 100, 539-553.

02 McBride, S. J., Williams, R. W., Creason, J., 2007. Bayesian hierarchical modeling of personal exposure to particulate matter. Atmospheric Environment 41 (29), $6143-6155$.

04 Meliker, J., Sloan, C., 2011. Spatio-temporal epidemiology: Principles and opportunities. Spatial and Spatio-temporal Epidemiology 2, 1-9.

905 Mitchell, T., Beauchamp, J., 1988. Bayesian variable selection in linear regression. Journal of the American Statistical Association 83, $1023-1032$.

906 Molitor, J., Molitor, N.-T., Jerrett, M., McConnell, R., Gauderman, J., Berhane, K., Thomas, D., 2006. Bayesian modeling of air pollution health effects with missing exposure data. American Journal of Epidemiology 164 (1), 69-76.

08 Morgenstern, H., 1998. Encyclopedia of Biostatistics. Vol. 2. JohnWiley \& Sons, New York, Ch. Ecologic study, pp. $1255-1276$.

909 Nuckols, J. R., Ward, M. H., Jarup, L., 06 2004. Using geographic information systems for exposure assessment in environmental epidemiology studies. Environmental Health Perspectives 112 (9), 1007-1015.

Openshaw, S., Taylor, P., 1981. Quantitative geography. London. Routledge, Ch. The modifiable areal unit problem.

12 Osei, F., 2014. Current statistical methods for spatial epidemiology: A review. Austin Biometrics and Biostatistics 1 (2), 1-7.

913 Ozkaynak, H., Baxter, L. K., Dionisio, K. L., Burke, J., 2013. Air pollution exposure prediction approaches used in air pollution epidemiology studies. Journal of Exposure Science and Environmental Epidemiology 23 (6), 566-572.

15 Paci, L., Gelfand, A., Cocchi, D., 2015. Quantifying uncertainty for temperature maps derived from computer models. Spatial Statistics 12, 96-108.

Paci, L., Gelfand, A., Holland, M., 2013. Spatio-temporal modeling for real-time ozone forecasting. Spatial Statistics 4, 79-93.

17 Paciorek, C. J., 2010. The importance of scale for spatial-confounding bias and precision of spatial regression estimators. Statistical science: a review journal of the Institute of Mathematical Statistics 25 (1), 107-125.

19 Peng, R., Dominici, F., 2008. Statistical Methods for Environmental Epidemiology with R. A Case Study in Air Pollution and Health. Springer.

Peng, R. D., Bell, M. L., 2010. Spatial misalignment in time series studies of air pollution and health data. Biostatistics (Oxford, England) 11 (4), $720-740$. 
Peng, R. D., Dominici, F., Louis, T. A., 2006. Model choice in time series studies of air pollution and mortality. Journal of the Royal Statistical Society: Series A (Statistics in Society) 169 (2), 179-203.

924 Peng, R. D., Dominici, F., Pastor-Barriuso, R., Zeger, S. L., Samet, J. M., 2005. Seasonal Analyses of Air Pollution and Mortality in 100 US Cities. American Journal of Epidemiology 161 (6), 585-594.

Peng, R. D., Dominici, F., Welty, L. J., 2009. A bayesian hierarchical distributed lag model for estimating the time course of risk of hospitalization associated with particulate matter air pollution. Journal of the Royal Statistical Society: Series C (Applied Statistics) 58 (1), 3-24.

Piantadosi, S., Byar, D., Green, S., 1988. The ecological fallacy. American Journal of Epidemiology 127 (5), 893-904.

Pinto Junior, J. A., Gamerman, D., Paez, M. S., Fonseca Alves, R. H., 2015. Point pattern analysis with spatially varying covariate effects, applied to the study of cerebrovascular deaths. Statistics in Medicine 34 (7), 1214-1226.

Pirani, M., Best, N., Blangiardo, M., Liverani, S., Atkinson, R. W., Fuller, G. W., 2015. Analysing the health effects of simultaneous exposure to physical and chemical properties of airborne particles. Environment International 79, 56 - 64.

Plummer, M., Clayton, D., 1996. Estimation of population exposure in ecological studies. Journal of the Royal Statistical Society, Series B 58, $113-126$.

35 Reich, B., Fuentes, M., Burke, J., 2008. Analysis of the effects of ultrafine particulate matter while accounting for human exposure. Environmetrics 20 (2), 131-146.

Reich, B. J., Hodges, J. S., Zadnik, V., 2006. Effects of residual smoothing on the posterior of the fixed effects in disease-mapping models. Biometrics 62 (4), 1197-1206.

Robert, C., Casella, G., 2004. Monte Carlo Statistical Methods. Springer.

Robertson, C., Nelson, T. A., MacNab, Y. C., Lawson, A. B., 2010. Review of methods for space-time disease surveillance. Spatial and Spatiotemporal Epidemiology 1 (2-3), 105 - 116, GEOMED Conference.

Rothman, K., Lash, T., Greenland, S., 2012. Modern Epidemiology, 3rd Edition. Wolters Kluwer.

Rue, H., Held, L., 2005. Gaussian Markov Random Fields. Theory and Applications. Chapman \& Hall.

Rue, H., Martino, S., Chopin, N., 2009. Approximate Bayesian inference for latent Gaussian models by using integrated nested Laplace approximations. Journal of the Royal Statistical Society: Series B (Statistical Methodology) 71 (2), 319-392.

Rue, H., Riebler, A., Sørbye, S. H., Illian, J. B., Simpson, D. P., Lindgren, F. K., 2016. Bayesian Computing with INLA: A Review. ArXiv e-prints. URL http: //adsabs.harvard.edu/abs/2016arXiv160400860R

Rushworth, A., Lee, D., Mitchell, R., 2014. A spatio-temporal model for estimating the long-term effects of air pollution on respiratory hospital admissions in Greater London. Spatial and Spatio-temporal Epidemiology 10, 29-38.

Sahu, S., Gelfand, A., Holland, D., 2006. Spatio-temporal modeling of fine particulate matter. Journal of Agricultural, Biological and Environmental Statistics 11, 61-86.

Sahu, S., Mardia, K., 2005. A Bayesian Kriged-Kalman model for short-term forecasting of air pollution level. Journal of the Royal Statistical Society, Series C 54, 223-244.

Sain, S., Cressie, N., 2007. A spatial model for multivariate lattice data. Journal of Econometrics 140, $226-259$.

Sain, S., Furrer, R., Cressie, N., 2011. A spatial analysis of multivariate output from regional climate models. The Annals of Applied Statistics 5, $150-175$.

Samet, M., Zeger, S., Dominici, F., Curriero, F., Coursac, I., Dockery, D., Schwartz, J., Zanobetti, A., 2000. The National Morbidity, Mortality, and Air Pollution Study. Tech. rep., Research Report: Health Effect Institute, Cambridge, MA.

Samoli, E., Touloumi, G., Zanobetti, A., Le Tertre, A., Schindler, C., Atkinson, R., Vonk, J., Rossi, G., Saez, M., Rabczenko, D., Schwartz, J., Katsouyanni, K., 2003. Investigating the dose-response relation between air pollution and total mortality in the aphea-2 multicity project. Occupational and Environmental Medicine 60 (12), 977-982.

963 Schikowski, T., Sugiri, D., Ranft, U., Gehring, U., Heinrich, J., Wichmann, H.-E., Kramer, U., 2005. Long-term air pollution exposure and living close to busy roads are associated with COPD in women. Respiratory Research 6 (1), 152.

Schmidt, A., Gelfand, A., 2003. A bayesian coregionalization model for multivariate pollutant data. Journal of Geophysics Research 108 (494), 8783.

67 Schwartz, J., Marcus, A., 1990. Mortality and air pollution in London: a time series analysis. American Journal of Epidemiology 131 (1), $185-194$. 68 Seeger, M., 2008. Bayesian inference and optimal design for the sparse linear model. The Journal of Machine Learning Research 9, $759-813$.

969 Shaddick, G., Zidek, J., 2014. A case study in preferential sampling: Long term monitoring of air pollution in the UK. Spatial Statistics 9, 51-65. 970 Shaddick, G., Zidek, J., 2015. Spatio-Temporal Methods in Environmental Epidemiology. Chapman \& Hall.

971 Sheppard, L., Burnett, R., Szpiro, A., Kim, S., Jerrett, M., Pope, C., Brunekreef, B., 2012. Confounding and exposure measurement error in air 972 pollution epidemiology. Air Quality, Atmosphere \& Health 5 (2), 203-216.

973 Smith, R., Davis, J., Sacks, J., Speckman, P., Styer, P., 2000. Regression models for air pollution and daily mortality: Analysis of data from 974 Birmingham, Alabama. Environmetrics 11, 719-734.

975 Smith, R., Kolenikov, S., 2003. Spatiotemporal modeling of PM 2.5 data with missing values. Journal of Geophysical Research 108 (D24), 11-1,11$976 \quad 11$.

977 Stern, H., Cressie, N., 2000. Posterior predictive model checks for disease mapping models. Statistics in Medicine 19, $2377-97$.

978 Szpiro, A., Sheppard, L., Lumley, T., 2011. Efficient measurement error correction with spatially misaligned data. Biostatistics 12 (4), 610-623.

979 Szpiro, A. A., Paciorek, C. J., 2013. Measurement error in two-stage analyses, with application to air pollution epidemiology. Environmetrics 24 (8), 501-517.

981 Tibshirani, R., 1996. Regression shrinkage and selection via the lasso. Journal of the Royal Statistical Society: Series B 58, 267-288.

982 Ugarte, M., Etxeberria, J., Goicoa, T., Ardanaz, E., 2012. Gender-specific spatiotemporal patterns of colorectal cancer incidence in Navarre, Spain (1990-2005). Cancer Epidemiology 36 (3), 254-62.

984 Valentini, P., Ippoliti, L., Fontanella, L., 2013. Modeling us housing prices by spatial dynamic structural equation models. The Annals of Applied 985 Statistics 7, 763-798.

986 Van de Kassteele, J., Stein, A., 2006. A model for external drift kriging with uncertain covariates applied to air quality measurements and dispersion 
model output. Environmetrics 17, 309-322.

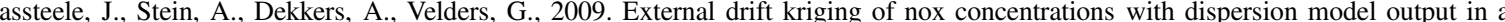
reduced air quality monitoring network. Environ Ecol Stat 16 (3), 321-339.

Wakefield, J., 2007. Disease mapping and spatial regression with count data. Biostatistics 8 (2), $158-183$.

Wakefield, J., Elliot, P., 1999. Issues in statistical analysis of small area health data. Statistics in Medicine 18, $2377-2399$.

Wakefield, J., Lyons, H., 2010. Handbook of Spatial Statistics. Chapman \& Hall, Ch. Spatial aggregation and the ecological fallacy.

Wakefield, J., Shaddick, G., 2006. Health-exposure modeling and the ecological fallacy. Biostatistics 7 (3), $438-455$.

96 Waller, L., Gotway, C., 2004. Applied Spatial Statistics for Public Health Data. Wiley.

997 West, M., 2003. Bayesian factor regression models in the "Large p, Small n" paradigm. Bayesian Statistics 7, 723-732.

998 WHO, 2015. Economic cost of the health impact of air pollution in Europe: Clean air, health and wealth. Tech. rep., WHO Regional Office for Europe, http://www.euro.who.int/en/media-centre/events/events/2015/04/ehp-mid-term-review/publications/ economic-cost-of-the-health-impact-of-air-pollution-in-europe.

Wikle, C., Berliner, L., 2005. Combining information across spatial scales. Technometrics 47 (1), 80-91.

Williams, P., 1995. Bayesian regularization and pruning using a Laplace prior. Neural Computation 7, $117-143$.

Young, L. J., Gotway, C. A., Yang, J., Kearney, G., DuClos, C., 2009. Linking health and environmental data in geographical analysis: It's so much more than centroids. Spatial and Spatio-temporal Epidemiology 1 (1), $73-84$.

Zhang, Y., Hodges, J., Banerjee, S., 2009. Smoothed ANOVA with spatial effects as a competitor to MCAR in multivariate spatial smoothing. Annals of Applied Statistics 3 (4), 1805-1830.

Zhu, L., Carlin, B. P., Gelfand, A., 2003. Hierarchical regression with misaligned spatial data: relating ambient ozone and pediatric asthma ER visits in Atlanta. Environmetrics 14 (5), 537-557.

Zidek, J., Shaddick, G., Taylor, C., 2014. Reducing estimation bias in adaptively changing monitoring nteworks with preferential site selection. The Annals of Applied Statistics 8 (3), 1640-1670.

Zidek, J., Sun, L., Le, N., Ozkaynak, H., 2002. Contending with space-time interaction in the spatial prediction of pollution: Vancouver's hourly ambient $\mathrm{PM}_{10}$ field. Environmetrics 13, 595-613.

Zidek, J. V., Shaddick, G., Meloche, J., Chatfield, C., White, R., 2007. A framework for predicting personal exposures to environmental hazards. Environmental and Ecological Statistics 14 (4), 411-431.

015 Zou, H., Hastie, T., Tibshirani, R., 2006. Sparse principal component analysis. Journal of Computational and Graphical Statistics 1, 265-286. 